\title{
Immunotherapy in Combination with Well-Established Treatment Strategies in Pancreatic Cancer: Current Insights
}

\author{
Christo Kole $\mathbb{D}^{1}$, Nikolaos Charalampakis $\mathbb{D}^{2}$, Sergios Tsakatikas ${ }^{2}$, Maximos Frountzas ${ }^{3}$, \\ Konstantinos Apostolou (1)', Dimitrios Schizas (1D)
}

'First Department of Surgery, National and Kapodistrian University of Athens, Laikon General Hospital, Athens, II5 27, Greece; ${ }^{2}$ Department of Medical Oncology, Metaxa Cancer Hospital, Athens, 185 37, Greece; ${ }^{3}$ First Department of Propaedeutic Surgery, National and Kapodistrian University of Athens, Hippocration General Hospital, Athens, II5 27, Greece

Correspondence: Dimitrios Schizas, First Department of Surgery, National and Kapodistrian University of Athens, Laikon General Hospital, Athens, II5 27, Greece, Tel +3069445059I7, Fax+302I3206I766, Email schizasad@gmail.com

\begin{abstract}
Pancreatic ductal adenocarcinoma (PDAC) is the most common type of pancreatic cancer and fourth most common cause of death in developed countries. Despite improved survival rates after resection combined with adjuvant chemotherapy or neoadjuvant chemotherapy, recurrence still occurs in a high percentage of patients within the first 2 years after resection. Immunotherapy aims to improve antitumor immune responses and reduce toxicity providing a more specific, targeted therapy compared to chemotherapy and has been proved an efficient therapeutic tool for many solid tumors. In this work, we present the latest advances in PDAC treatment using a combination of immunotherapy with other interventions such as chemotherapy and/or radiation both at neoadjuvant and adjuvant setting. Moreover, we outline the role of the tumor microenvironment as a key barrier to immunotherapy efficacy and examine how immunotherapy biomarkers may be used to detect immunotherapy's response.
\end{abstract}

Keywords: pancreatic cancer, immunotherapy, immune checkpoint inhibitors, cancer vaccines, adoptive cellular immunotherapy, microsatellite instability

\section{Introduction}

Pancreatic ductal adenocarcinoma (PDAC) is the most common type of pancreatic cancer accounting for more than $90 \%$ of $\operatorname{cases}^{1}$ (commonly referred to as pancreatic cancer), other types being cystadenocarcinoma and acinar cell carcinoma. ${ }^{2}$ PDAC represents a substantial health problem as presented on GLOBOCAN 2020 with as many deaths $(466,000)$ as cases $(496,000)$, affecting both sexes equally and rating pancreatic cancer as the seventh leading cause of cancer death globally. ${ }^{3}$ Furthermore, especially in developed countries, PDAC is the fourth most common cause of death, however, the disease is predicted to reach second place within the next decade. ${ }^{4}$ PDAC is associated with an extremely poor prognosis with less than $5 \%$ of the patients reaching 5-year overall survival (OS) $)^{5,6}$ due to late diagnosis, rapid tumor progression and limited available treatments. ${ }^{7}$

Novel approaches, such as immunotherapy, aim to improve antitumor immune responses providing a more specific targeted therapy compared to chemotherapy. In recent years, immunotherapy has established its role in the treatment of various solid tumors including PDAC ${ }^{8-10}$ Immune checkpoint inhibitors (ICIs) are monoclonal antibodies used to block extracellular proteins expressed by the tumour or tumor-associated lymphocytes resulting in the suppression of antitumor immune response. ${ }^{11}$ Examples of such extracellular proteins which prevent the $\mathrm{T}$ cells from recognizing and eliminating cancer cells are, the programmed cell death protein-1 (PD-1), and its ligand PD-L1 and cytotoxic T-lymphocyte-associated antigen 4 (CTLA-4) ${ }^{12}$ PD-1 binds to PD-L1 and PD-L2 ligands therefore preventing T-cell activity in peripheral tissues. ${ }^{13}$ CTLA-4 is another inhibitory receptor that modulates the initial stages of T-cell activation and prevents immune hyperactivation by competitively inhibiting the binding of B7 ligands to the co-stimulatory receptor Cluster Differentiation 28 (CD-28). ${ }^{13-16}$ Both PD-L1 and CTLA-4 are overexpressed in a subset of PDACs and are associated with worse survival, ${ }^{17}$ therefore consisting of 
a promising therapeutic target. Furthermore, other strategies such as cancer vaccines use tumor-associated antigens (TAAs) to trigger immunization by activating the cytotoxic T-lymphocytes (CTLs). Tumor-associated antigens are derived either from whole-cell tumor lysates, recombinant tumor peptides or recombinant viruses. ${ }^{18-20}$ On the other hand, adoptive cell transfer (ACT) includes genetically engendered autologous immune cells from patients' peripheral blood. These cells bind to specific cancer antigens leading to the immune destruction of tumor cells (Figure 1). ${ }^{21}$ In addition, the chemokine system, a key regulator for inflammatory responses and leukocyte trafficking with chemokine receptor 2 (CCR2) and chemokine receptor 5 (CCR5), is playing an important role in this process; ${ }^{22,23}$ CCR2 expression is relatively restricted to certain cell types, monocytes, NK and T lymphocytes, and it is shown to have a strong proinflammatory function, predominantly expressed by monocytes/macrophages, while when expressed in tumor microenvironments, it can be strongly immunosuppressive. ${ }^{24}$ Therefore, targeting the recruitment of immunosuppressive monocytes/macrophages in tumors by CCR2 antagonism is used as a strategy to modify the tumor microenvironment and enhance anti-tumor immunity. ${ }^{25}$ Despite the robust results in certain malignancies, neither immune checkpoint inhibitors nor vaccines or adoptive cell transfer have yet shown promising clinical efficacy in PDAC when used as single agent in most phases I and II clinical trials. ${ }^{9,26,27}$ On the other hand a combination of immunotherapy with chemoradiation regimens have shown encouraging results. The aim of our work is to present a state-of-the-art review of the latest advances in PDAC treatment using a combination of immunotherapy with other strategies such as chemotherapy and radiation therapy both in the neoadjuvant and adjuvant setting.

\section{Neoadjuvant Therapy}

The majority of patients with PDAC are diagnosed in late stage disease with the tumour being either unresectable or metastatic. Only $15 \%$ to $20 \%$ are candidates for immediate tumor resection. ${ }^{28}$ Adjuvant chemotherapy is considered the

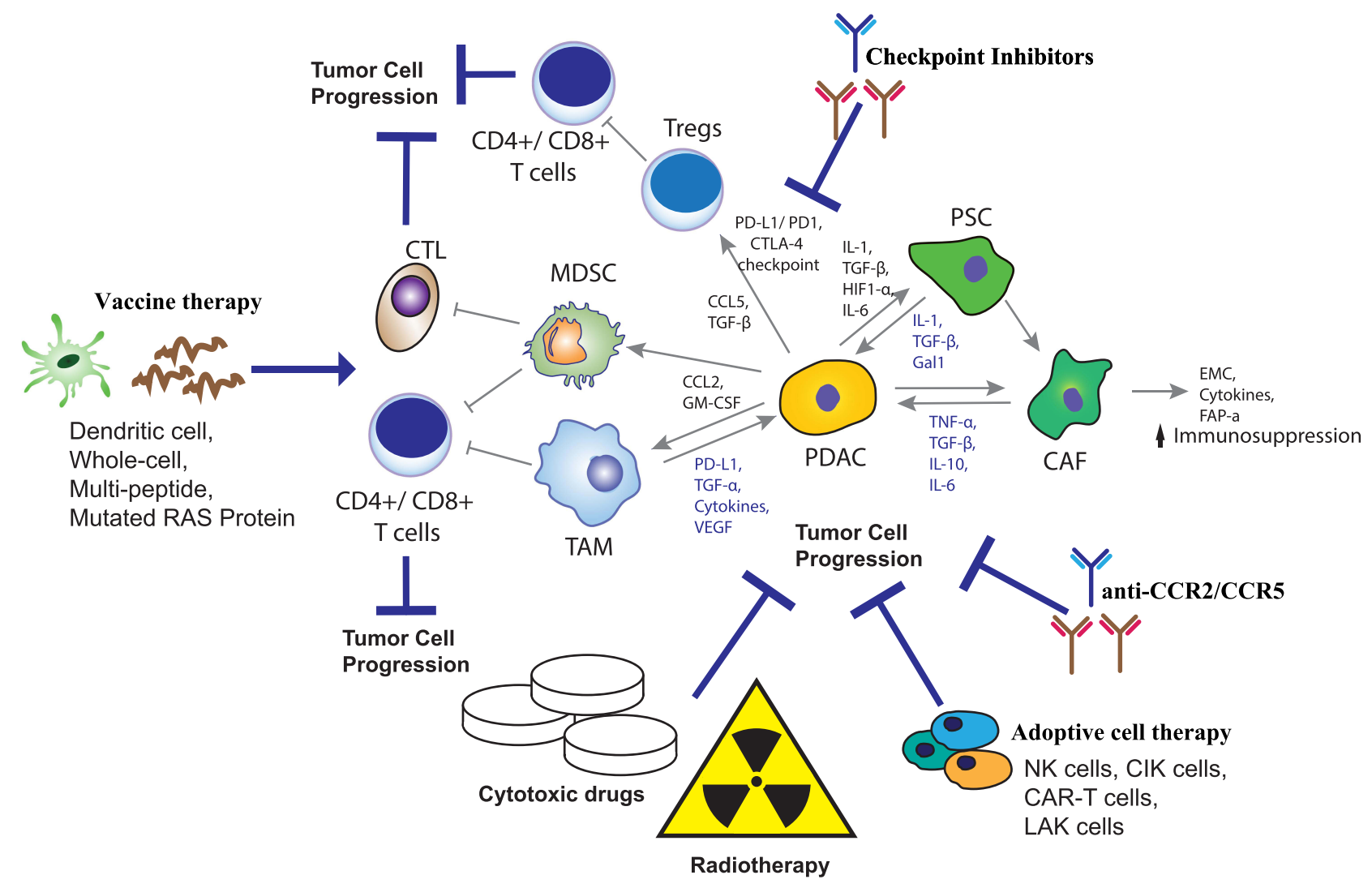

Figure I Therapeutic strategies target components of the tumor microenvironment in PDAC. Tumor microenvironment creates a barrier for immunotherapy and cytotoxic drugs in pancreatic cancer. Inhibition of the resistance mechanisms in pancreatic cancer by vaccine therapy and checkpoint inhibitors lead in activation of T-cells and tumor cell destruction. Adoptive cell transfer (ACT) lead to immune destruction of tumor cells while cytotoxic drugs and radiotherapy improve the efficacy of anticancer therapy. Adapted with permission from Schizas D, Charalampakis N, Kole C, et al. Immunotherapy for pancreatic cancer: a 2020 update. Cancer Treat Rev. 2020;86:I020I6. ${ }^{9}$

Abbreviations: ECM, extracellular matrix; PDAC, pancreatic adenocarcinoma; CAF, carcinoma- associated fibroblasts; MDSC, myeloid-derived suppressive cells; TAM, tumor-associated macrophages; CTL, cytotoxic T-lymphocytes; PSC, pancreatic stellate cells; CCL2/CCR2, C-C motif chemokine ligand/receptor. 
current standard-of-care after surgical resection of PDAC. ${ }^{29}$ However, recurrence still occurs in more than $75 \%$ of patients within the first 2 years after resection probably due to micrometastases, ${ }^{30}$ increasing the need for optimization of neoadjuvant strategies and the development of new drug targets. ${ }^{28}$ Neoadjuvant therapy is a systemic treatment prior to definitive surgical therapy which is supposed to provide an improved local and distant control. ${ }^{31}$ According to the current clinical practice guidelines for pancreatic cancer, ${ }^{29}$ neoadjuvant therapy is indicated for patients with borderline resectable and locally advanced PDAC, whereas its role for resectable PDAC is still under investigation. Immunotherapy was associated with tumor regression and improved OS in preclinical studies of PDAC when used in combination with other treatments. ${ }^{32}$ However, there was no significant difference in the median OS for neoadjuvant or adjuvant immunotherapy. Nevertheless, in the neoadjuvant subset analysis, immunotherapy was associated with significantly improved OS compared to no immunotherapy. ${ }^{33}$ Moreover, neoadjuvant immunotherapy may increase the presence of TILs in the PC microenvironment ${ }^{34}$ and reduce the immunosuppressive effects of surgery by systemic release of glucocorticoids which induce apoptosis of naïve T cells and suppression of T-cell proliferation. ${ }^{35,36}$ Together these limit the probability of recurrence. ${ }^{34}$ Several clinical trials using a combination of immunotherapy and chemotherapy or chemoradiotherapy in the neoadjuvant setting are currently ongoing (Table 1). In a retrospective analysis studying the impact of neoadjuvant and adjuvant immunotherapy in the survival of pancreatic cancer patients, no significant difference in OS between neoadjuvant and adjuvant immunotherapy was found (HR: 1.06, 95\% confidence interval (CI): 0.79-1.41; $\mathrm{p}<0.714$ ). However, multivariable Cox regression analysis revealed that neoadjuvant immunotherapy was associated with significantly improved OS (HR: $0.86,95 \%$ CI: $0.74-0.99 ; p<0.04$ ) compared to no immunotherapy only in patients with a high-level of education, but not in patients with a low-level of education. ${ }^{33}$ This difference between populations may be due to the fact that high-level of education patients are more prone to stick to the therapeutic plan and follow doctor instructions compared to low-level of education patients.

In phase Ib/II trial (NCT02305186), Rahma et al reported that pembrolizumab, an immune checkpoint inhibitor (antiPD-1 IgG4 antibody), in combination with capecitabine and radiation as neoadjuvant regimen resulted in increased median recurrence free survival (RFS), 18.2 vs 14.1 months $(p=0.41)$, and overall survival $(\mathrm{OS}), 27.8$ vs 24.3 months $(\mathrm{p}=0.68)$, compared to chemoradiation alone (Table 1). ${ }^{37}$ Moreover, the authors reported that $70 \%$ of the patients treated with pembrolizumab plus chemoradiation compared to $53 \%$ receiving chemoradiation alone underwent surgery. ${ }^{37}$ This study is currently enrolling 25 more patients who will receive FOLFIRINOX prior to randomization to CRT \pm pembrolizumab, which will help to further investigate the immune modulatory effect of chemotherapy followed by CRT. FOLFIRINOX is also used as a neoadjuvant chemotherapy backbone in the NCT03983057 phase 3 trial in patients with locally advanced or borderline resectable PDAC. Contrary to the previous trial, this study evaluates chemoimmunotherapy without the addition of preoperative radiation. ${ }^{38}$

Immunotherapy agents have been successfully combined with other targeted therapies in several cancer types. Preclinical data suggest the existence of a synergistic effect between immunotherapy and certain targeted agents such as antiangiogenic factors and TKIs. ${ }^{39}$ An ongoing phase II study examines the efficacy of the focal adhesion kinase (FAK) inhibitor defactinib in combination with pembrolizumab in patients with PDAC in the perioperative setting. FAK is a protein tyrosine kinase which is frequently overexpressed in various malignancies. FAK inhibition affects several intracellular pathways associated with cell survival and is involved in the immunomodulation of tumor microenvironment, ${ }^{40}$ thus representing a promising therapeutic partner for immunotherapy regimens. Accordingly, a phase I safety trial in patients with various solid tumors, including PDAC has also produced encouraging safety and efficacy results when defactinib was combined with pembrolizumab. ${ }^{41}$

Likewise, treatment options have been significantly expanded with the addition of cancer vaccines and adoptive cell therapy, which use either cancer related antigens to sensitize the host's immune system against tumor cells, or in-vitro enhance patient's lymphocytes to identify and target tumor cells. ${ }^{42}$ Since the efficacy of checkpoint inhibitors in PDAC seems limited, at least based on available results from completed clinical trials, attention has partially shifted towards the development of effective cancer vaccines. GVAX is a whole tumor cell vaccine genetically engineered to express granulocyte-macrophage colony-stimulating factor (GM-CSF). ${ }^{43}$ The efficacy of GVAX in combination with CY has been studied as neoadjuvant therapy in stage I/II (resectable or borderline resectable) pancreatic cancer (Table 1). ${ }^{44}$ Combination with CY resulted in lower disease free survival (DFS) and OS compared to GVAX monotherapy; ie 8.54 
Table I Adjuvant Immunotherapy in Surgically Resected Pancreatic Adenocarcinoma

\begin{tabular}{|c|c|c|c|c|c|c|}
\hline Intervention & & Cancer Stage & $\begin{array}{l}\text { Clinical } \\
\text { Phasel } \\
\text { Identifier }\end{array}$ & $\begin{array}{l}\text { Outcomes: Disease } \\
\text { Free Survival } \\
\text { (Months) }(95 \% \mathrm{Cl})\end{array}$ & $\begin{array}{l}\text { Outcomes: Median } \\
\text { Overall Survival } \\
\text { (Months) }(95 \% \mathrm{Cl})\end{array}$ & Reference \\
\hline $\begin{array}{l}\text { GVAX/5-FU/ } \\
\text { radiation }\end{array}$ & $\begin{array}{l}\text { Whole-cell vaccine, } \\
\text { Cytotoxic drugs }\end{array}$ & $\begin{array}{l}\text { Resected stage I or II } \\
\text { pancreatic } \\
\text { adenocarcinoma }\end{array}$ & $\begin{array}{l}\text { Phase II, } \\
\text { NCT00084383 }\end{array}$ & $17.3(14.6-22.8)$ & $24.8(21.2-31.6)$ & $\begin{array}{l}\text { Lutz et al } \\
2011^{60}\end{array}$ \\
\hline $\begin{array}{l}\text { GVAXI } \\
\text { Chemoradiation }\end{array}$ & $\begin{array}{l}\text { Whole-cell vaccine, } \\
\text { Cytotoxic drugs }\end{array}$ & $\begin{array}{l}\text { Stage II or III Positive/ } \\
\text { nevative margins }\end{array}$ & Phase I & 13 & 20 & $\begin{array}{l}\text { Jaffee et al } \\
2001^{61}\end{array}$ \\
\hline $\begin{array}{l}\text { Algenpantucel-L/ } \\
\text { Chemoradiation }\end{array}$ & $\begin{array}{l}\text { Multi-peptide vaccine, } \\
\text { Chemoradiation }\end{array}$ & $\begin{array}{l}\text { Surgically Resected } \\
\text { Pancreatic Cancer }\end{array}$ & $\begin{array}{l}\text { Phase II, } \\
\text { NCT00569387 }\end{array}$ & $62 \%$ I2-month DFS & $86 \%$ 12-month OS & $\begin{array}{l}\text { Hardacre, } \\
\text { Mulcahy } \\
\text { et al } 2013^{62}\end{array}$ \\
\hline $\begin{array}{l}\text { Algenpantucel-L, } \\
\text { Gemcitabine/5-FU/ } \\
\text { Rradiation }\end{array}$ & $\begin{array}{l}\text { Multi-peptide vaccine, } \\
\text { Cytotoxic drugs, radiation }\end{array}$ & $\begin{array}{l}\text { Surgically resected } \\
\text { pancreatic cancer }\end{array}$ & $\begin{array}{l}\text { Phase III, } \\
\text { NCT0I07298I }\end{array}$ & $\begin{array}{l}\text { Completed } \\
\text { No results posted }\end{array}$ & $\begin{array}{l}\text { Completed } \\
\text { No results posted }\end{array}$ & $\begin{array}{l}\text { McCormick } \\
\text { et al } 2016^{64}\end{array}$ \\
\hline $\begin{array}{l}\text { K-Ras vaccine/GM- } \\
\text { CSF }\end{array}$ & $\begin{array}{l}\text { Peptide vaccine, } \\
\text { Costimulatory molecule }\end{array}$ & $\begin{array}{l}\text { Surgically resected } \\
\text { and advanced disease } \\
\text { patients }\end{array}$ & $\begin{array}{l}\text { Phase I/II } \\
\text { CTNRAS95002 } \\
\text { CTNRAS97004 }\end{array}$ & No results posted & $\begin{array}{l}25.6(10-39) \text { for Res } \\
10.2(3-28) \text { for NonRes }\end{array}$ & $\begin{array}{l}\text { Gjertsen } \\
\text { et al } 2001^{66}\end{array}$ \\
\hline $\begin{array}{l}\text { Ras-peptide/GM- } \\
\text { CSF/Chemotherapy }\end{array}$ & $\begin{array}{l}\text { Peptide vaccine, } \\
\text { Costimulatory molecules, } \\
\text { chemotherapy }\end{array}$ & $\begin{array}{l}\text { Resected KRAS } \\
\text { mutant pancreatic } \\
\text { cancer }\end{array}$ & NA & No results posted & $20.3(95 \% \mathrm{Cl}, \mathrm{II} .6-45.3)$ & $\begin{array}{l}\text { Abou-Alfa } \\
\text { et al } 2011^{67}\end{array}$ \\
\hline $\begin{array}{l}\text { TG0I/GM-CSF } \\
\text { /Gemcitabine }\end{array}$ & $\begin{array}{l}\text { Vaccine, Costimulatory } \\
\text { molecules, chemotherapy }\end{array}$ & $\begin{array}{l}\text { Resected pancreatic } \\
\text { cancer }\end{array}$ & $\begin{array}{l}\text { Phase I/II } \\
\text { NCT0226I7I4 }\end{array}$ & $16.1(11.1-19.6)$ & $33.3(24.0-40.0)$ & ASA $2012^{68}$ \\
\hline $\begin{array}{l}\text { Gl-4000/ } \\
\text { Gemcitabine } \\
\text { Placebo/ } \\
\text { Gemcitabine }\end{array}$ & $\begin{array}{l}\text { Mutated RAS Protein } \\
\text { vaccine, Cytotoxic drugs }\end{array}$ & $\begin{array}{l}\text { Resected pancreatic } \\
\text { cancer/ KRAS mutant }\end{array}$ & Phase II & $\begin{array}{l}9.4 \\
8.3\end{array}$ & $\begin{array}{l}17.22 \\
14.5\end{array}$ & $\begin{array}{l}\text { Muscarella } \\
\text { et al } 2012^{69}\end{array}$ \\
\hline $\begin{array}{l}\text { Nivolumab/GVAX/ } \\
\text { CRS-207/CY } \\
\text { GVAX/CRS-207/CY }\end{array}$ & $\begin{array}{l}\text { PD-I inhibitor, Whole-cell } \\
\text { vaccine, Listeria vaccine, } \\
\text { Cytotoxic drugs }\end{array}$ & $\begin{array}{l}\text { Previously treated } \\
\text { metastatic pancreatic } \\
\text { adenocarcinoma }\end{array}$ & $\begin{array}{l}\text { Phase II, } \\
\text { NCT0224337I }\end{array}$ & $\begin{array}{l}2.23(2.14-2.33) \\
2.17(2.00-2.30)\end{array}$ & $\begin{array}{l}5.88(4.73-8.64) \\
6.11(3.52-7.00)\end{array}$ & $\begin{array}{l}\text { Hopkins } \\
\text { et al } 2015^{77}\end{array}$ \\
\hline $\begin{array}{l}\text { KIF20A/VEGFRI/ } \\
\text { VEGFR2/ } \\
\text { Gemcitabine }\end{array}$ & $\begin{array}{l}\text { Peptide cocktail vaccine, } \\
\text { Cytotoxic drugs }\end{array}$ & $\begin{array}{l}\text { Resected pancreatic } \\
\text { cancer patients }\end{array}$ & Phase II & $15.8(11.1-20.6)$ & NR & $\begin{array}{l}\text { Miyazawa } \\
\text { et al } 2017^{70}\end{array}$ \\
\hline $\begin{array}{l}\text { Nivolumab, MS- } \\
813160, \text { GVAX }\end{array}$ & $\begin{array}{l}\text { PD-I inhibitor, CCR2/ } \\
\text { CCR5 dual antagonist, } \\
\text { Whole-cell vaccine }\end{array}$ & $\begin{array}{l}\text { Locally advanced } \\
\text { pancreatic cancer }\end{array}$ & $\begin{array}{l}\text { Phase I/II, } \\
\text { NCT03767582 }\end{array}$ & Recruiting & Recruiting & $\begin{array}{l}\text { Hopkins } \\
2019^{71}\end{array}$ \\
\hline $\begin{array}{l}\text { Nivolumab, MS- } \\
813160, \\
\text { Gemcitabine, Nab- } \\
\text { paclitaxel }\end{array}$ & $\begin{array}{l}\text { PD-I inhibitor, CCR2/ } \\
\text { CCR5 dual antagonist, } \\
\text { Cytotoxic drugs }\end{array}$ & $\begin{array}{l}\text { Resectable and locally } \\
\text { advanced pancreatic } \\
\text { cancer }\end{array}$ & $\begin{array}{l}\text { Phase I/II, } \\
\text { NCT03496662 }\end{array}$ & Recruiting & Recruiting & $\begin{array}{l}\text { Medicine } \\
\text { WUSo et al } \\
2018^{72}\end{array}$ \\
\hline
\end{tabular}

Abbreviations: 5-FU, fluorouracil; GM-CSF, granulocyte-macrophage colony-stimulating factor; CY, cyclophosphamide; KIF20A, kinesin family member 20A; VEGFRI/R2, vascular endothelial growth factor receptor 1/2; Cl, confidence interval; Res, resected; NonRes, non-resected; NR, not reached; NA, not applicable.

months (95\% CI, 2.66-17.1) compared to 18.92 months (95\% CI, 13.87-34.1). OS was also lower, 15.4 months $(95 \%$ CI, 13.2-26.5) compared to 34.2 months (95\% CI, 21.6-45.7) respectively. ${ }^{44}$ Another ongoing phase 2 study combined GVAX with pembrolizumab and SBRT in 58 patients with locally advanced PDAC. Among them, 35 patients underwent surgery and the median distant metastases free for the entire cohort reached 9.7 months, ${ }^{45}$ while a phase 2 study in a similar population combines GVAX with pembrolizumab, cyclophosphamide and SBRT $^{46}$ with results still pending. The NCT03153410 early Phase 1 trial adds IMC CS4, a monoclonal antibody blocking the colony-stimulating factor receptor, to the aforementioned combination in patients with borderline resectable PDAC. ${ }^{47}$ Additional strategies have 
been employed including a combination of cancer vaccines, checkpoint inhibitors and chemoradiation to increase the efficacy of neoadjuvant therapy, however, no results have been reported so far. ${ }^{48-54}$ On the other hand, the safety and efficacy of CCX872-B, a CCR2 Inhibitor, is currently evaluated in patients with histologically or cytologically confirmed non-resectable pancreatic adenocarcinoma with or without metastases also receiving FOLFIRINOX (NCT02345408). ${ }^{55}$ Another phase $1 \mathrm{~b}$ trial (NCT03778879) was designed to evaluate the efficacy of CCX872-B in combination with stereotactic body radiotherapy for preoperative treatment of resectable pancreatic cancer, however, the study was discontinued due to an insufficient quantity of the CCX872-B drug to conduct the study. ${ }^{56}$ Furthermore, another phase I trial (NCT01413022) ${ }^{57}$ studies the side effects and optimal dose of PF-04136309 (a CCR2 inhibitor) in combination with chemotherapy (FOLFIRINOX) in patients with locally advanced or borderline resectable pancreatic cancer, who are not candidates for surgical resection. During this study, no treatment-related deaths occurred while grade 3 or higher adverse events were reported in at least $10 \%$ of the patients receiving PF-04136309. Six out of 33 (49\%) patients receiving FOLFIRINOX plus PF-04136309 who had undergone repeat imaging achieved an objective tumor response, while in the FOLFIRINOX alone group, none of the five patients with repeat imaging achieved an objective response. ${ }^{58}$

\section{Adjuvant Therapy}

Since surgery alone is associated with short-term survival and early tumor relapse, the implementation of systemic therapy is needed in the majority of patients. ${ }^{59}$ A phase II clinical study in resected, stage I or II pancreatic adenocarcinoma patients, showed an increased DFS of 17.3 months (95\% CI, 14.6-22.8), and OS of 24.8 (95\% CI, 21.2-31.6) when patients were treated with whole-cell vaccine GIVAX in combination with chemoradiation in an adjuvant setting (Table 2). ${ }^{60}$ (Table 2) These findings were also supported by Jaffee et al, although this was a phase I trial performed in stage II or III pancreatic cancer patients with both positive and negative resection margins, and so was not designed to test clinical benefit. ${ }^{61}$ A phase 2 multi-institutional trial, evaluated the use of algenpantucel-L immunotherapy in addition to chemotherapy and chemoradiotherapy in the adjuvant setting for resected pancreatic

Table 2 Neoadjuvant Immunotherapy in Resectable or Borderline Resectable Pancreatic Adenocarcinoma

\begin{tabular}{|c|c|c|c|c|c|c|}
\hline Intervention & & Cancer Stage & $\begin{array}{l}\text { Clinical } \\
\text { Phasel } \\
\text { Identifier }\end{array}$ & $\begin{array}{l}\text { Outcomes: } \\
\text { Disease Free } \\
\text { Survival (Months) } \\
(95 \% \mathrm{Cl})\end{array}$ & $\begin{array}{l}\text { Outcomes: } \\
\text { Median Overall } \\
\text { Survival (Months) } \\
(95 \% \mathrm{Cl})\end{array}$ & Reference \\
\hline $\begin{array}{l}\text { Pembrolizumab/ } \\
\text { Capecitabine/Radiation } \\
\text { Capecitabine/Radiation }\end{array}$ & $\begin{array}{l}\text { PD-I inhibitor, } \\
\text { Cytotoxic drugs, }\end{array}$ & $\begin{array}{l}\text { Resectable or } \\
\text { Borderline } \\
\text { Resectable } \\
\text { Adenocarcinoma }\end{array}$ & $\begin{array}{l}\text { Phase Ib/ll, } \\
\text { NCT02305I86 }\end{array}$ & $\begin{array}{c}18.2 \\
14.1(p 0.41)\end{array}$ & $\begin{array}{c}27.8 \\
24.3(p 0.68)\end{array}$ & $\begin{array}{c}\text { Rahma et al. } \\
2021^{37}\end{array}$ \\
\hline $\begin{array}{l}\text { Pembrolizumab/ } \\
\text { Defactinib }\end{array}$ & $\begin{array}{l}\text { PD-I inhibitor, Kinase } \\
\text { inhibitor }\end{array}$ & $\begin{array}{l}\text { Resectable } \\
\text { Adenocarcinoma }\end{array}$ & $\begin{array}{l}\text { Phase II, } \\
\text { NCT03727880 }\end{array}$ & Recruiting & Recruiting & $\begin{array}{l}\text { Hopkins et al } \\
2019^{41}\end{array}$ \\
\hline $\begin{array}{l}\text { Pembrolizumab/GVAXI } \\
\text { IMC-CS4/CY }\end{array}$ & $\begin{array}{l}\text { PD-I inhibitor, Whole- } \\
\text { cell vaccine, } \\
\text { Antineoplastic agent, } \\
\text { Cytotoxic drug }\end{array}$ & $\begin{array}{l}\text { Borderline } \\
\text { Resectable } \\
\text { Adenocarcinoma }\end{array}$ & $\begin{array}{l}\text { Early Phase I, } \\
\text { NCT03I534I0 }\end{array}$ & $\begin{array}{l}\text { Active, not } \\
\text { recruiting }\end{array}$ & $\begin{array}{l}\text { Active, not } \\
\text { recruiting }\end{array}$ & $\begin{array}{c}\text { Hopkins et al } \\
2018^{47}\end{array}$ \\
\hline $\begin{array}{l}\text { GVAX/CY } \\
\text { GVAX }\end{array}$ & $\begin{array}{l}\text { Whole-cell vaccine, } \\
\text { Cytotoxic drugs }\end{array}$ & $\begin{array}{l}\text { Resectable or } \\
\text { Borderline } \\
\text { Resectable } \\
\text { Adenocarcinoma }\end{array}$ & $\begin{array}{l}\text { Phase II, } \\
\text { NСT0072744I }\end{array}$ & $\begin{array}{c}8.54(2.66-17.1) \\
18.92(13.87-34.1)\end{array}$ & $\begin{array}{l}15.4(13.2-26.5) \\
34.2(21.6-45.7)\end{array}$ & Hopkins $2008^{44}$ \\
\hline $\begin{array}{l}\text { Nivolumab/GVAX/CY/ } \\
\text { Urelumab }\end{array}$ & $\begin{array}{l}\text { PD-I inhibitor, Whole- } \\
\text { cell vaccine, Cytotoxic } \\
\text { drugs, Antineoplastic } \\
\text { agent }\end{array}$ & $\begin{array}{l}\text { Resectable } \\
\text { Adenocarcinoma }\end{array}$ & $\begin{array}{l}\text { Phase I, II, } \\
\text { NCT02451982 }\end{array}$ & Recruiting & Recruiting & $\begin{array}{l}\text { Hopkins et al } \\
2016^{51}\end{array}$ \\
\hline
\end{tabular}

(Continued) 
Table 2 (Continued).

\begin{tabular}{|c|c|c|c|c|c|c|}
\hline Intervention & & Cancer Stage & $\begin{array}{l}\text { Clinical } \\
\text { Phasel } \\
\text { Identifier }\end{array}$ & $\begin{array}{l}\text { Outcomes: } \\
\text { Disease Free } \\
\text { Survival (Months) } \\
(95 \% \mathrm{Cl})\end{array}$ & $\begin{array}{l}\text { Outcomes: } \\
\text { Median Overall } \\
\text { Survival (Months) } \\
(95 \% \mathrm{Cl})\end{array}$ & Reference \\
\hline $\begin{array}{l}\text { Nivolumab/FOLFRINOX } \\
\text { (FFX) }\end{array}$ & $\begin{array}{l}\text { PD-I inhibitor, } \\
\text { chemotherapy regimen }\end{array}$ & $\begin{array}{l}\text { Borderline } \\
\text { Resectable } \\
\text { Adenocarcinoma }\end{array}$ & $\begin{array}{l}\text { Phase I/2, } \\
\text { NCT03970252 }\end{array}$ & Recruiting & Recruiting & $\begin{array}{l}\text { Center, Squibb } \\
\text { et al } 2019^{52}\end{array}$ \\
\hline $\begin{array}{l}\text { Nivolumab/GVAX/BMS- } \\
813160 / \text { SBRT }\end{array}$ & $\begin{array}{l}\text { PD-I inhibitor, Whole- } \\
\text { cell vaccine, } \\
\text { Antineoplastic agent, } \\
\text { Radiation }\end{array}$ & $\begin{array}{l}\text { Locally Advanced } \\
\text { Adenocarcinoma }\end{array}$ & $\begin{array}{l}\text { Phase I/2, } \\
\text { NCT03767582 }\end{array}$ & Recruiting & Recruiting & Hopkins $2019^{54}$ \\
\hline $\begin{array}{l}\text { GMCl/Chemotherapy/ } \\
\text { Radiation }\end{array}$ & $\begin{array}{l}\text { Adenoviral vector, } \\
\text { Chemoradiation }\end{array}$ & $\begin{array}{l}\text { Advanced Non- } \\
\text { Metastatic } \\
\text { Pancreatic } \\
\text { Adenocarcinoma }\end{array}$ & $\begin{array}{l}\text { Phase I/2, } \\
\text { NCT02446093 }\end{array}$ & Recruiting & Recruiting & $\begin{array}{l}\text { Candel } \\
\text { Therapeutics and } \\
\text { University } 2015^{53}\end{array}$ \\
\hline $\begin{array}{l}\text { M7824/ } \\
\text { M924I/ } \\
\text { SBRT }\end{array}$ & $\begin{array}{l}\text { PD-LI inhibitor, } \\
\text { Immunocytokine }\end{array}$ & $\begin{array}{l}\text { Advanced Pancreas } \\
\text { Cancer }\end{array}$ & $\begin{array}{l}\text { Phase } 1 / 2 \text {, } \\
\text { NCT04327986 }\end{array}$ & Recruiting & Recruiting & $\begin{array}{l}\text { Institute and } \\
\text { Center } 2021^{48}\end{array}$ \\
\hline $\begin{array}{l}\text { Algenpantucel-L/ } \\
\text { FOLFRINOX/ } \\
\text { Gemcitabine/5-FU }\end{array}$ & $\begin{array}{l}\text { Multi-peptide vaccine, } \\
\text { Cytotoxic drugs }\end{array}$ & $\begin{array}{l}\text { Borderline } \\
\text { resectable } \\
\text { pancreatic cancer }\end{array}$ & $\begin{array}{l}\text { Phase II, } \\
\text { NCT02405585 }\end{array}$ & $\begin{array}{l}\text { Study terminated } \\
\text { No results posted }\end{array}$ & $\begin{array}{l}\text { Study terminated } \\
\text { No results posted }\end{array}$ & $\begin{array}{c}\text { Corporation and } \\
\text { Pharma } 2015^{49}\end{array}$ \\
\hline $\begin{array}{l}\text { Algenpantucel-L/ } \\
\text { FOLFRINOX/ } \\
\text { Gemcitabine/Nab- } \\
\text { paclitaxel/Capecitabine/ } \\
\text { 5-FU }\end{array}$ & $\begin{array}{l}\text { Multi-peptide vaccine, } \\
\text { Cytotoxic drugs }\end{array}$ & $\begin{array}{l}\text { Resectable (stage } \\
\text { II), Unresectable } \\
\text { (stage III) }\end{array}$ & $\begin{array}{l}\text { Phase III, } \\
\text { NCT0I836432 }\end{array}$ & $\begin{array}{l}\text { Study terminated } \\
\text { No results posted }\end{array}$ & $\begin{array}{l}\text { Study terminated } \\
\text { No results posted }\end{array}$ & $\begin{array}{l}\text { Corporation and } \\
\text { Pharma } 2013^{50}\end{array}$ \\
\hline CCX872-B/FOLFIRINOX & $\begin{array}{l}\text { CCR2 Inhibitor, } \\
\text { Cytotoxic drugs }\end{array}$ & $\begin{array}{l}\text { Patients With } \\
\text { Pancreatic } \\
\text { Adenocarcinoma }\end{array}$ & $\begin{array}{l}\text { Phase Ib, } \\
\text { NCT02345408 }\end{array}$ & $\begin{array}{l}\text { Active, not } \\
\text { recruiting }\end{array}$ & No results posted & ChemoCentryx ${ }^{55}$ \\
\hline CCX872-B/SBRT & $\begin{array}{l}\text { CCR2 Inhibitor, } \\
\text { Radiation }\end{array}$ & $\begin{array}{l}\text { Resectable } \\
\text { pancreatic cancer }\end{array}$ & $\begin{array}{l}\text { Phase Ib, } \\
\text { NCT03778879 }\end{array}$ & $\begin{array}{l}\text { Withdrawn } \\
\text { No results posted }\end{array}$ & No results posted & $\begin{array}{l}\text { Katz and } \\
\text { Rochester } \\
2019^{56}\end{array}$ \\
\hline $\begin{array}{l}\text { PF-04I36309/ } \\
\text { FOLFIRINOX }\end{array}$ & $\begin{array}{l}\text { CCR2 Inhibitor, } \\
\text { Cytotoxic drugs }\end{array}$ & $\begin{array}{l}\text { Borderline } \\
\text { resectable and } \\
\text { locally advanced } \\
\text { pancreatic cancer }\end{array}$ & $\begin{array}{l}\text { Phase I, } \\
\text { NCT0I4I3022 }\end{array}$ & $\begin{array}{l}\text { CCR2-targeted } \\
\text { therapy plus } \\
\text { FOLFIRINOX is safe } \\
\text { and tolerable }\end{array}$ & & $\begin{array}{l}\text { Nywening et al } \\
2016^{58} \\
\text { Medicine, } \\
\text { W. U. S. o., and } \\
\text { Institute, N. C. }{ }^{57}\end{array}$ \\
\hline
\end{tabular}

Abbreviations: CY, cyclophosphamide; FOLFIRINOX (FFX), oxaliplatin, leucovorin, irinotecan, 5-fluorouracil; SBRT, stereotactic body radiation; GMCI, gene mediated cytotoxic immunotherapy (aglatimagene besadenovec plus valacyclovir); 5-FU, fluorouracil; $\mathrm{Cl}$, confidence interval.

cancer, resulted in $62 \% 12$-month disease-free survival and $86 \% 12$-month overall survival. ${ }^{62}$ However, encouraging results from this phase II trial did not translate into clinical benefit in the phase III IMPRESS clinical trial, where the addition of algenpantucel-L to chemotherapy did not improve OS in patients with resected PDAC. Algenpantucel-L has also failed to prove its effectiveness in the treatment of locally advanced PDAC in the PILLAR phase III trial, ${ }^{63}$ Nevertheless, phase III trial assessing overall survival after treatment with Algenpantucel-L, chemotherapy and radiation is completed however no results have been reported so far. ${ }^{64}$

The majority of pancreatic adenocarcinoma patients have mutations in KRAS oncogene. ${ }^{65}$ In order to enhance the immune response, synthetic mutant RAS peptides plus GM-CSF were used as adjuvant treatment in a phase I/II trial, leading in peptide-specific immunity induction in $58 \%$ of the patients. ${ }^{66}$ Patients that responded to the peptide vaccine 
had higher OS (4.9 months) compared to non-responders (2 months). Another study, however, yielded low immune response ( 1 out of 9 patients) and a median OS of 20.3 months. ${ }^{67}$ TG01 is injectable antigen-specific cancer immunotherapy which induces RAS-mutant-specific T-cell responses, enhanced by co-administration of GM-CSF. Patients with stage I or II pancreatic adenocarcinoma (R0 or R1) receiving adjuvant gemcitabine with TG01/GM-CSF presented a median OS of 33.1 (95\% CI 24.0-40.0) months, and median DFS of 16.1 months (95\% CI, 11.1-19.6) ${ }^{68}$ (Table 2). Improved outcomes are also observed after an inactivated recombinant saccharomyces cerevisiae expressing mutant RAS protein (GI-4000) combined with gemcitabine in an adjuvant setting. ${ }^{69}$ The phase II clinical study showed improvement in survival in R1 subjects with Ras mutant positive pancreas cancer and a significantly higher rate of mutation specific T cell response. The median OS was reported at 17.2 and 14.5 months and RFS at 9.4 and 8.3 months for GI-4000 group and placebo group respectively. ${ }^{69}$ In addition, peptide cocktail vaccine (OCV-C01) plus gemcitabine in patients with resected pancreatic cancer presented a median DFS at 15.8 (95\% CI, 11.1-20.6) months with OS while OS at 18 months was $69 \%$ (95\% CI, 48.8-82.5). ${ }^{70}$ In addition, all four patients who underwent R0 resection with KIF20A expression had no recurrence of pancreatic cancer with KIF20A-specific CTL responses. ${ }^{70}$ Concerning CCL2/CCR2 Axis, a phase I/II study (NCT03767582) was established to evaluate the combination of nivolumab and a CCR2/CCR5 dual antagonist (BMS-813160) with GVAX in patients with locally advanced pancreatic cancer (LAPC) who have received chemotherapy and radiotherapy. Safety profile is checked as well as whether this combination therapy enhances the infiltration of CD8+CD137+ cells in PDACs. ${ }^{71}$ Another phase I/II study (NCT03496662) which is currently under recruitment status evaluates the tolerability and efficacy of bms- 813160 with nivolumab, gemcitabine and nab-paclitaxel in borderline resectable and locally advanced PDAC. ${ }^{72}$

\section{Advanced Metastatic}

The majority of patients with PDAC are diagnosed at late stages with the tumor being either unresectable or metastatic. ${ }^{28}$ For patients with locally advanced and metastatic pancreatic cancer the treatment of choice is palliative chemotherapy, although very few improvements are seen in survival. ${ }^{29,59}$ However, recent combination therapies including immunotherapy have shown some promising outcomes (Table 3). A phase Ib clinical trial in unresectable (Stage III) or metastatic pancreatic cancer patients treated with CTLA-4 inhibitor, ipilimumab, showed improved outcomes when ipilimumab was combined with gemcitabine. ${ }^{73}$ Patients who received ipilimumab at $6 \mathrm{mg} / \mathrm{kg}$ in weeks $1,4,7$ and 10 and gemcitabine hydrochloride at $1, \mathrm{mg} / \mathrm{m}^{2}$ in weeks $1-7$ and $9-11$, showed 3.86 months (95\% CI, 0.75 to 22.41$)$ and 8.99 months (95\% CI, 0.75-30.04) of progression free survival (PFS) and OS respectively. ${ }^{73}$ In another study, a combination of ipilimumab with a PD-1 inhibitor, nivolumab, and MEK inhibitor, cobimetinib (arm 2), reported improved objective response rate (ORR) compared to ipilimumab plus nivolumab (arm 1), at 6.7 (95\% CI, 0.8-22.1) versus 0.0 (95\% CI, 0.0-18.5) months respectively. ${ }^{74}$ Nevertheless, patients in arm 2 were treated at $3 \mathrm{mg} / \mathrm{kg}$ nivolumab plus cobimetinib, compared to arm 1 where nivolumab was used at $1 \mathrm{mg} / \mathrm{kg} .{ }^{75}$ Ipilimumab also improved OS when combined with GVAX, administrated in locally advanced or metastatic pancreatic cancer, resulting in OS of 5.7 months (95\% CI, 4.3-14.7) compared to 3.6 months (95\% CI, 2.5-9.2) when used as monotherapy. ${ }^{76}$ On the other hand, a combination of nivolumab and GIVAX, listeria vaccine CRS-207 and CY, resulted in no survival differences compared to GIVAX/CRS-207/CY in patients with previously treated metastatic pancreatic adenocarcinoma. ${ }^{77}$ These poor results may be due to the increased progression of metastatic pancreatic adenocarcinoma and therefore failure of treatment.

The use of tremelimumab, another CTLA-4 inhibitor in combination with PD-1 inhibitor, durvalumab, or gemcitabine showed modest but higher OS and DFS compared to tremelimumab or durvalumab monotherapy in advanced/metastatic tumors (Table 3). ${ }^{27,78}$ However, no differences were observed when tremelimumab plus durvalumab were used in metastatic adenocarcinoma patients with progression after fluoropyrimidine or gemcitabine first-line chemotherapy. ${ }^{27}$ Another study performed in advanced or metastatic pancreatic cancer patients treated with pembrolizumab plus gemcitabine and Nab-paclitaxel resulted in improved PFS and OS at 9.1 months (95\% CI, 4.9-13/3) and 15.0 months (95\% CI, 6.8-22.6) respectively. ${ }^{79,80}$ Further clinical trials using PD-1 inhibitors in combination with chemotherapies are currently ongoing. The NCT03977272 trial uses the anti-PD-1 immune checkpoint inhibitor camrelizumab in combination with FOLFIRINOX in patients with metastatic PDAC that have received either no previous treatment or only gemcitabine-based first-line chemotherapy. ${ }^{81}$ Another single arm study evaluated the safety and efficacy of camrelizumab 
Table 3 Clinical Trials in Locally Advanced or Metastatic Pancreatic Adenocarcinoma

\begin{tabular}{|c|c|c|c|c|c|c|}
\hline Intervention & & Cancer Stage & $\begin{array}{l}\text { Clinical Phase/ } \\
\text { Identifier }\end{array}$ & $\begin{array}{l}\text { Outcomes: } \\
\text { Disease Free } \\
\text { Survival } \\
\text { (Months) } \\
(95 \% \mathrm{Cl})\end{array}$ & $\begin{array}{l}\text { Outcomes: } \\
\text { Median Overall } \\
\text { Survival } \\
\text { (Months) } \\
(95 \% \mathrm{Cl})\end{array}$ & Reference \\
\hline $\begin{array}{l}\text { Ipilimumab/ } \\
\text { Gemcitabine }\end{array}$ & $\begin{array}{l}\text { CTLA-4 inhibitor, } \\
\text { Cytotoxic drugs }\end{array}$ & $\begin{array}{l}\text { Unresectable (Stage III/ IV) or } \\
\text { metastatic pancreatic cancer }\end{array}$ & $\begin{array}{l}\text { Phase Ib, } \\
\text { NCTOI473940 }\end{array}$ & $3.8(0.7-22.4)$ & $8.9(0.7-30.0)$ & $\begin{array}{l}\text { University and } \\
\text { Center } 2012^{73}\end{array}$ \\
\hline $\begin{array}{l}\text { Ipilimumab/ } \\
\text { Nivolumab/ } \\
\text { Cobimetinib }\end{array}$ & $\begin{array}{l}\text { CTLA-4 inhibitor, } \\
\text { PD-I inhibitor, MEK } \\
\text { inhibitor }\end{array}$ & Advanced/metastatic Tumor & $\begin{array}{l}\text { Phase I/II, } \\
\text { NCT0I928394 }\end{array}$ & \multicolumn{2}{|c|}{ ORR 6.7 ( $95 \% \mathrm{Cl} 0.8-22.1)$ months } & Squibb $2013^{75}$ \\
\hline $\begin{array}{l}\text { Ipilimumab/ } \\
\text { GVAX } \\
\text { Ipilimumab }\end{array}$ & $\begin{array}{l}\text { CTLA-4 inhibitor, } \\
\text { Whole-cell vaccine }\end{array}$ & $\begin{array}{l}\text { Locally advanced, unresectable } \\
\text { or metastatic pancreatic }\end{array}$ & $\begin{array}{l}\text { Phase I, } \\
\text { NCT00836407 }\end{array}$ & No results posted & $\begin{array}{l}5.7(4.3-14.7) \\
3.6(2.5-9.2)\end{array}$ & Le et al $2013^{76}$ \\
\hline $\begin{array}{l}\text { Tremelimumab/ } \\
\text { Durvalumab } \\
\text { Tremelimumab } \\
\text { Durvalumab }\end{array}$ & $\begin{array}{l}\text { CTLA-4 inhibitor, } \\
\text { PD-I inhibitor }\end{array}$ & Advanced/Metastatic Tumor & $\begin{array}{l}\text { Phase II, } \\
\text { NCT02527434 }\end{array}$ & $\begin{array}{l}2.86(1.87-3.52) \\
1.77(1.38-2.92) \\
1.84(1.84-1.84)\end{array}$ & $\begin{array}{l}7.18(3.98-18.76) \\
3.98(2.83-5.13) \\
4.14 \text { (NA-NA) }\end{array}$ & AstraZeneca $2015^{27}$ \\
\hline $\begin{array}{l}\text { Tremelimumab/ } \\
\text { Durvalumab } \\
\text { Durvalumab }\end{array}$ & $\begin{array}{l}\text { CTLA-4 inhibitor, } \\
\text { PD-I inhibitor }\end{array}$ & $\begin{array}{l}\text { Metastatic adenocarcinoma/ } \\
\text { progression after } \\
\text { fluoropyrimidine or gemcitabine } \\
\text { first-line chemotherapy }\end{array}$ & $\begin{array}{l}\text { Phase II, } \\
\text { NCT02558894 }\end{array}$ & $\begin{array}{l}1.5(1.2-1.5) \\
1.5(1.3-1.5)\end{array}$ & $\begin{array}{l}3.1(2.2-6.1) \\
3.6(2.7-6.1)\end{array}$ & AstraZeneca $2015^{27}$ \\
\hline $\begin{array}{l}\text { Tremelimumab/ } \\
\text { Gemcitabine }\end{array}$ & $\begin{array}{l}\text { CTLA-4 inhibitor, } \\
\text { Cytotoxic drugs }\end{array}$ & $\begin{array}{l}\text { Advanced Metastatic Pancreatic } \\
\text { Cancer }\end{array}$ & $\begin{array}{l}\text { Phase I, } \\
\text { NCT00556023 }\end{array}$ & No results posted & $7.4(5.8-9.4)$ & Aglietta et al $2014^{78}$ \\
\hline $\begin{array}{l}\text { Pembrolizumab/ } \\
\text { Gemcitabine/ } \\
\text { Nab-paclitaxel }\end{array}$ & $\begin{array}{l}\text { PD-I inhibitor, } \\
\text { Cytotoxic drugs }\end{array}$ & $\begin{array}{l}\text { Advanced Metastatic Pancreatic } \\
\text { Cancer }\end{array}$ & $\begin{array}{l}\text { Phase Ib/ll, } \\
\text { NCT0233|25I }\end{array}$ & $9.1(4.9-13 / 3)$ & $15.0(6.8-22.6)$ & $\begin{array}{l}\text { Weiss et al } 2019^{79} \\
\text { Weiss et al } 2018^{80}\end{array}$ \\
\hline $\begin{array}{l}\text { Nivolumab/ } \\
\text { OSE2I0I/ } \\
\text { FOLFIRI }\end{array}$ & $\begin{array}{l}\text { PD-I inhibitor, } \\
\text { multi-neoepitope } \\
\text { vaccine, Cytotoxic } \\
\text { drugs }\end{array}$ & Locally Advanced or Metastatic & $\begin{array}{l}\text { Phase 2, } \\
\text { NCT03806309 }\end{array}$ & Recruiting & Recruiting & $\begin{array}{l}\text { Group, } \\
\text { Immunotherapeutics } \\
\text { et al } 2019^{148}\end{array}$ \\
\hline $\begin{array}{l}\text { Atezolizumab, } \\
\text { KYIO44 }\end{array}$ & $\begin{array}{l}\text { PD-LI inhibitor, } \\
\text { Inducible T cell co- } \\
\text { stimulator }\end{array}$ & $\begin{array}{l}\text { Advanced Metastatic Pancreatic } \\
\text { Cancer }\end{array}$ & $\begin{array}{l}\text { Phase I/II, } \\
\text { NCT0382950I }\end{array}$ & Recruiting & Recruiting & $\begin{array}{l}\text { Limited and Sanofi } \\
2019^{149}\end{array}$ \\
\hline $\begin{array}{l}\text { GVAX/CRS- } \\
207 / C Y \\
\text { GVAX/CY }\end{array}$ & $\begin{array}{l}\text { Whole-cell vaccine, } \\
\text { Listeria vaccine, } \\
\text { Cytotoxic drugs }\end{array}$ & $\begin{array}{l}\text { Metastatic pancreatic } \\
\text { adenocarcinoma }\end{array}$ & $\begin{array}{l}\text { Phase } 2 \mathrm{a} \text {, } \\
\text { NCT0I4I7000 }\end{array}$ & No results posted & $\begin{array}{l}6.28(4.47-9.40) \\
4.07(3.32-5.42)\end{array}$ & Whiting et al $2015^{91}$ \\
\hline $\begin{array}{l}\text { GVAX/CRS- } \\
207 / C Y \\
\text { CRS-207 } \\
\text { CY }\end{array}$ & $\begin{array}{l}\text { Whole-cell vaccine, } \\
\text { Listeria vaccine, } \\
\text { Cytotoxic drugs }\end{array}$ & $\begin{array}{l}\text { Previously treated metastatic } \\
\text { pancreatic adenocarcinoma }\end{array}$ & $\begin{array}{l}\text { Phase Ilb, } \\
\text { NCT02004262 }\end{array}$ & No results posted & $\begin{array}{l}3.7(2.9-5.3) \\
5.4(4.2-6.4) \\
4.6(4.2-5.7)\end{array}$ & Le et al $2019^{92}$ \\
\hline $\begin{array}{l}\text { KIF20A-66 } \\
\text { BSC }\end{array}$ & Peptide vaccine & $\begin{array}{l}\text { Metastatic pancreatic } \\
\text { adenocarcinoma, previously } \\
\text { treated with gemcitabine }\end{array}$ & $\begin{array}{l}\text { Phase I/II, } \\
\text { UMIN000004919 }\end{array}$ & No results posted & $\begin{array}{l}4.7 \pm 0.8 \\
2.7 \pm 1.1\end{array}$ & Asahara et al $2013^{93}$ \\
\hline $\begin{array}{l}\text { KIF20A/ } \\
\text { VEGFRI/ } \\
\text { VEGFR2/ } \\
\text { Gemcitabine }\end{array}$ & $\begin{array}{l}\text { Peptide vaccine, } \\
\text { Peptide vaccine, } \\
\text { Peptide vaccine, } \\
\text { Cytotoxic drugs }\end{array}$ & $\begin{array}{l}\text { Advanced and/or metastatic } \\
\text { pancreatic cancer }\end{array}$ & $\begin{array}{l}\text { Phase II, } \\
\text { UMIN000008082 }\end{array}$ & $\begin{array}{l}\text { 4.7, HLA-matched } \\
5.2, \text { HLA- } \\
\text { unmatched }\end{array}$ & $\begin{array}{l}\text { 9.0, HLA-matched } \\
\text { 10.0, HLA- } \\
\text { unmatched }\end{array}$ & Suzuki et al $2017^{94}$ \\
\hline
\end{tabular}

(Continued) 
Table 3 (Continued).

\begin{tabular}{|c|c|c|c|c|c|c|}
\hline Intervention & & Cancer Stage & $\begin{array}{l}\text { Clinical Phase/ } \\
\text { Identifier }\end{array}$ & $\begin{array}{l}\text { Outcomes: } \\
\text { Disease Free } \\
\text { Survival } \\
\text { (Months) } \\
\text { (95\% Cl) }\end{array}$ & $\begin{array}{l}\text { Outcomes: } \\
\text { Median Overall } \\
\text { Survival } \\
\text { (Months) } \\
(95 \% \mathrm{Cl})\end{array}$ & Reference \\
\hline $\begin{array}{l}\text { MUCI, HLA- } \\
\text { A2, ICAM-I, } \\
\text { LFA-3, GM-CSF }\end{array}$ & $\begin{array}{l}\text { Vaccinia virus-tumor } \\
\text { antigens, } \\
\text { Costimulatory } \\
\text { molecules }\end{array}$ & $\begin{array}{l}\text { Unresectable or metastatic } \\
\text { pancreatic cancer }\end{array}$ & Phase I & No results posted & $\begin{array}{l}\text { I5.3, anti CEA/ } \\
\text { MUC-I positive } \\
3.9 \text {, anti CEA/ } \\
\text { MUC-I negative }\end{array}$ & Kaufman et al $2007^{95}$ \\
\hline $\begin{array}{l}\text { w/ Poly-ICLC } \\
\text { plus peptide- } \\
\text { pulsed DC-CIK }\end{array}$ & $\begin{array}{l}\text { Immunostimulant } \\
\text { DC-CIK vaccination }\end{array}$ & $\begin{array}{l}\text { Metastatic, unresectable } \\
\text { pancreatic cancer }\end{array}$ & $\begin{array}{l}\text { Phase I } \\
\text { NCT0I410968 }\end{array}$ & No results posted & 7.7 months & $\begin{array}{l}\text { Mehrotra et al } \\
2017^{150}\end{array}$ \\
\hline $\begin{array}{l}\text { DC-CIK } \\
\text { /Chemotherapy } \\
\text { S-I } \\
\text { DC-CIK } \\
\text { Chemotherapy } \\
\text { S-I } \\
\text { BSC }\end{array}$ & $\begin{array}{l}\text { DC-CIK } \\
\text { vaccination, } \\
\text { Cytotoxic drugs }\end{array}$ & $\begin{array}{l}\text { Metastatic, unresectable } \\
\text { pancreatic cancer }\end{array}$ & $\begin{array}{l}\text { Phase I /II, } \\
\text { NCT0I78I520 }\end{array}$ & $\begin{array}{l}4.5 \\
\\
2.8 \\
3 \\
1.4\end{array}$ & $\begin{array}{l}7 \\
\\
4.2 \\
4.7 \\
1.73\end{array}$ & Jiang et al $2017^{97}$ \\
\hline $\begin{array}{l}\text { GVI00I/ } \\
\text { Gemcitabine/ } \\
\text { Capecitabine } \\
\text { Gemcitabine/ } \\
\text { Capecitabine }\end{array}$ & $\begin{array}{l}\text { Peptide vaccine, } \\
\text { Cytotoxic drugs }\end{array}$ & $\begin{array}{l}\text { Locally advanced or metastatic } \\
\text { pancreatic cancer }\end{array}$ & $\begin{array}{l}\text { Phase III, } \\
\text { NCT02854072 }\end{array}$ & No results posted & $\begin{array}{l}6.9(6.4-7.6) \\
7.9(7.1-8.8)\end{array}$ & $\begin{array}{l}\text { Middleton et al } \\
2014^{96}\end{array}$ \\
\hline $\begin{array}{l}\text { MORAb-009, } \\
\text { Gemcitabine } \\
\text { Placebo/ } \\
\text { Gemtacibine }\end{array}$ & $\begin{array}{l}\text { Monoclonal } \\
\text { antibody, Cytotoxic } \\
\text { drugs }\end{array}$ & Unresectable (stage III or IV) & $\begin{array}{l}\text { Phase II, } \\
\text { NCT005707I3 }\end{array}$ & No results posted & $\begin{array}{l}6.5(4.5-8.10) \\
6.9(5.4-8.8)\end{array}$ & Morphotek $2007^{151}$ \\
\hline
\end{tabular}

Abbreviations: FOLFIRI, fluorouracil, leucovorin, irinotecan, and oxaliplatin; BSC, best supportive care; CY, cyclophosphamide; KIF20A, kinesin family member 20A; VEGFRI/R2, vascular endothelial growth factor receptor I/2; MUCI, mucin I; ICAM-I, intercellular adhesion molecule I; LFA-3, lymphocyte function-associated antigen 3; GM-CSF, granulocyte-macrophage colony-stimulating factor; $\mathrm{Cl}$, confidence interval; ORR, objective response rate; NR, not reached.

in combination with gemcitabine and nab-paclitaxel in 20 patients with advanced PDAC. More than half of the study's population responded to the treatment regimen and almost all patients achieved disease control. Toripalimab is another anti PD-L1 antibody used as first line treatment in combination with nab-paclitaxel and gemcitabine as first-line treatment for advanced pancreatic adenocarcinoma in a phase Ib/II trial of 20 untreated patients with advanced PDAC. ORR was $35.3 \%$ and DCR $82.4 \%$ and PFS and OS reached 5 months and 14 months respectively. ${ }^{82}$ Results from another early phase trial of the PD-1 inhibitor sintilimab showed a similar efficacy rate and a predictable toxicity profile. ${ }^{83}$

The development of monoclonal antibodies targeting immune checkpoints in order to counter the immunosuppression of tumor microenvironment represents a significant development in the treatment of solid tumors and has significantly augmented the therapeutic armamentarium. This notion is further advanced with the development of bispecific proteins that bind to multiple receptor simultaneously and link tumor cells to lymphocytes, facilitating lymphocyte-mediated tumor cell destruction. ${ }^{84}$ Few trials are currently exploring bispecific molecules in patients with metastatic PDAC. Envafolimab is a bispecific PD-L1/CTLA-4 antibody currently tested in a phase II trial in combination with gemcitabine and nab-paclitaxel in the advanced setting. Preliminary results from 17 patients show a 55\% ORR and 88.9\% DCR, while around one-third of patients experienced grade 3 or higher treatment related adverse events, mainly hepatic toxicity and nausea. ${ }^{85}$

Another bispecific molecule tested in pretreated patients with mPDAC is the bispecific fusion protein targeting PD-L1 and the TGF- $\beta$ receptor. An ongoing phase II trial combines this novel molecule with the multi-potent TKI famitinib in patients with pretreated PDAC and preliminary safety data have not reported grade 4 or 5 toxicities till now. ${ }^{86}$ 
Cytokines are molecules that regulate intercellular interaction and play a major role in orchestrating the immune response to infections as well as to tumor cells. Use of cytokines such as interferon-alpha and interleukin-2 have been one of the first attempts to sensitize the host's immune system to tumor cells to induce lymphocyte mediated cell lysis. ${ }^{87}$ The optimal role of cytokines in cancer treatment is still being investigated in several clinical trials, especially in immunosensitive tumors such as melanoma and renal cell carcinoma. Since PDAC is relatively resistant to ICIs, attempts are being made to enhance their activity with the addition of cytokines in recent clinical trials. The phase II NCT04390763 study examines the safety and efficacy of the anti-TGF- $\beta$ monoclonal antibody NIS793 with and without the PD-1 inhibitor spartalizumab in combination with nab-paclitaxel and gemcitabine versus chemotherapy alone in patients with untreated metastatic PDAC ${ }^{88}$ Similarly, nadunolimab, an interleukin-1 receptor accessory protein inhibitor, was combined with chemotherapy in 36 untreated patients with advanced PDAC. ORR and DCR were 27\% and 57.6\% respectively, while mPFS reached 7.8 months. ${ }^{89}$ Unfortunately, the phase III SEQUOIA trial showed no benefit in OS or PFS with the addition of pegilodecakin, a pegylated IL-10 formation drug, to standard of care FOLFOX chemotherapy after progression on gemcitabine-based chemotherapy. ${ }^{90}$

Cancer vaccines have been used in several clinical studies (Table 3). The addition of listeria vaccine to GVAX plus CY resulted in improved OS compared to GVAX/CY regimen, 6.28 (95\% CI, 4.47-9.40) and 4.07 (95\% CI, 3.32-5.42) months respectively. ${ }^{91}$ On the other hand, the combination of Cy/GVAX + CRS-207 did not improve survival over chemotherapy as presented by a phase IIb trial (NCT02004262). ${ }^{92}$ A previously conducted phase I clinical trial combining the HLA-A*2402restricted KIF20A-derived peptide vaccine with gemcitabine for advanced pancreatic cancer confirmed its safety and immunogenicity increasing the OS compared to best supportive care (BSC) (Table 3) ${ }^{93}$ The VENUS-PC study, a singlearmed, phase II trial used two antiangiogenic cancer vaccines targeting VEGFR1 and VEGFR2 in addition to the KIF20A peptide. In this study, authors evaluated the clinical benefit of the cancer vaccination in combination with gemcitabine. ${ }^{94}$ No differences were observed either in median OS or in median PFS between the HLA-matched and HLA-unmatched group. ${ }^{94}$ Nevertheless, patients in the HLA-matched group with peptide-specific CTL induction for KIF20A or VEGFR1 had a better prognosis compared to those without such induction. ${ }^{94}$

Ten patients with advanced pancreatic cancer were treated in a phase I clinical trial with vaccination regimen consisted of vaccinia virus expressing tumor antigens carcinoembryonic antigen (CEA) and mucin-1 (MUC-1) with three costimulatory molecules B7.1, ICAM-1 and LFA-3 (TRICOM) (PANVAC-V) and fowlpox virus expressing the same antigens and costimulatory molecules (PANVAC-F).$^{95}$ Median overall survival was reported at 6.3 months with a significant increase in overall survival noted in patients who generated anti CEA- and/or MUC-1-specific immune responses compared with those who did not (15.1 vs 3.9 months, respectively; $\mathrm{P}=0.002$ ). Moreover, antibody responses were observed in all 10 patients while $62.5 \%$ of the patients had antigen-specific $\mathrm{T}$ cell responses. ${ }^{95}$ Another, open-label, randomised, phase 3 trial on locally advanced or metastatic pancreatic cancer patients using a combination of GV1001 peptide vaccine with gemcitabine and capecitabine failed to improve survival compared to chemotherapy alone. ${ }^{96} 358$ patients were allocated to the chemotherapy group, 350 to the sequential chemoimmunotherapy group, and 354 to the concurrent chemoimmunotherapy group. Median overall survival was reported at 7.9 months $(95 \% \mathrm{CI}, 7.1-8.8)$ for chemotherapy group, 6.9 months (95\% CI, 6.4-7.6) for sequential chemoimmunotherapy group and 8.4 months (95\% CI, 7.3-9.7) for concurrent chemoimmunotherapy group. ${ }^{96}$

On the other hand, immunostimulant DC-CIK vaccination in combination with the S-1 chemotherapy regimen in 47 patients with advanced pancreatic cancer resulted in favorable PFS and OS compared to monotherapy or best supportive care. ${ }^{97}$ Moreover, analysis of peripheral blood demonstrated that the $\mathrm{CD} 3^{+}, \mathrm{CD}^{+} / \mathrm{CD}^{+}$, and $\mathrm{CD} 8^{+} / \mathrm{CD} 28^{+} \mathrm{T}$-cell subsets were significantly elevated, while the $\mathrm{CD}^{+} / \mathrm{CD}^{+}, \mathrm{CD}^{+} / \mathrm{CD} 16^{+} / \mathrm{CD} 56^{+}$and $\mathrm{CD} 4^{+} / \mathrm{CD} 25^{+}$cell subsets were significantly decreased after DC-CIK cell therapy.

A recent breakthrough in adoptive cell therapy has been the development of chimeric antigen receptor (CAR) T-cells. These patient-derived lymphocytes have been genetically engineered and expanded ex-vivo to express receptors that target specific tumor antigens. ${ }^{98}$

When reinfused into patients, they cause tumor-cell destruction through multiple mechanisms, exploiting both the inhibition of tumor signaling pathways and the cytotoxic effect of T-cells. This new treatment modality has shown promising results, mainly in the treatment of hematologic malignancies. ${ }^{21}$ Several trials are currently exploring the efficacy and safety of CAR-T cells with various chimeric receptors in PDAC binding to already known tumor antigens 
such as Nectin4/FAP, mesothelin, HER2 and CEA, based on promising data from preclinical trial. ${ }^{99}$ A few clinical trials have already produced some early safety and efficacy results. ${ }^{100}$ A phase I trial of mesothelin targeting CAR-T cells tested in 6 patients with pretreated PDAC showed clinical benefit in 3 out of 6 patients NCT04037241, NCT03323944. ${ }^{101}$

Several novel molecules that target immune checkpoints have been developed and are being tested in the clinical setting. The combination of the novel CD40 agonist sotigalimab with gemcitabine and nab-paclitaxel with or without nivolumab produced some interesting results and showed promising efficacy in a phase 2 study. Interestingly though, the combination of sotigalimab and nivolumab led to worse outcomes than either of these drugs alone, suggesting a possible antagonistic effect among immunotherapy drugs. ${ }^{102}$

In addition to novel agents, other drugs that have been investigated or frequently used in different diseases have been employed in the treatment of PDAC. Masitinib is a TKI with activity against c-KIT, PDGFR and FGFR3 and has been investigated in the treatment of systemic mastocytosis due to its activity on mast cell and macrophage function, which may have an immunomodulatory effect on tumor microenvironment. ${ }^{103}$

The phase 3 AB12005 trial evaluated masitinib plus chemotherapy in 384 untreated patients with advanced or metastatic PDAC which suffered from cancer pain. Addition of masitinib improved PFS and OS in patients with locally advanced PDAC but not in the overall study population. ${ }^{104}$ The hypomethylating agent azacitidine is being investigated in a phase II trial NCT03264404 in combination with pembrolizumab in patients with pretreated PDAC. Finally, the phase I MEDIPLEX study is examining the TKI and colony-stimulating factor-1 receptor inhibitor pexidartinib, which is currently used for the treatment of tenosynovial giant cell tumor in combination with durvalumab in patients with advanced PDAC and colorectal cancer. ${ }^{105}$

The combination of chemotherapy and immunotherapy with local radiation has been explored as a preoperative approach in an attempt to downstage large invasive tumors and facilitate R0 resection. Exploiting the systemic biological synergy between immunotherapy and radiation, as suggested by several preclinical studies, ${ }^{106}$ a few trials have incorporated radiotherapy combinations in the advanced and metastatic setting. For example, the phase II/III QUILT 88 combines standard of care chemotherapy with low-dose chemoradiation and immunotherapy which consists of an infusion of NK-cells with high affinity for PD-L1 and an IL-15 cytokine fusion protein. ${ }^{107}$ Another phase II study uses camrelizumab in combination with chemotherapy and local ablation in patients with PDAC and liver metastases. ${ }^{108}$

\section{Immunotherapy Biomarkers in PDAC}

The tumor microenvironment (TME) acts as a physical barrier against effective delivery of systemic chemotherapy. ${ }^{109-111}$ Moreover, immune-suppressing mechanisms induced by regulatory T cells (Tregs), myeloid-derived suppressor cells (MDSC) and tumor-associated macrophages (TAM) accumulation, play a central role in suppressing antigen-presenting cells. Tregs lead in T-cell apoptosis and inhibit dendritic cell maturation and function, ${ }^{112}$ moreover, immune tolerance is also due to CTLA-4 which is constantly expressed on Tregs (Figure 1). ${ }^{113}$ On the other hand, MDSC inhibit both innate and adaptive antitumor immunity in pancreatic cancer ${ }^{114}$ while TAMs prevent dendritic cell-mediated antitumor immune responses by secreting a variety of growth factors. ${ }^{115,116}$ Moreover, the presence of regulatory (FOXP3+) T-cells prevents active T-cells through PD-1/ PD-L1 checkpoint inhibitor expression (Figure 1). ${ }^{117}$ Understanding of tumor microenvironments may lead in the identification of subgroups of patients who will have better outcome from certain therapies.

Accumulation of somatic mutations leads to increased neoantigen formation therefore renders tumors susceptible to immunotherapy through inflammatory cytokines and T-cell activation. ${ }^{118}$ The mismatch repair (MMR) system is a family of proteins (MLH1, MSH2, MSH3, MSH6, PMS1 and PMS2) playing an important role in error repair during DNA replication. Defects in this system (dMMR) leads to random mutations occurring in specific regions of short DNA sequences of repetitions (microsatellites), a mechanism known as microsatellite instability (MSI). ${ }^{119-121}$ In the majority of pancreatic cancer patients neoantigen formation is caused, however, due to the immunosuppressive tumor microenvironment, as effective immune responses fail to be generated. ${ }^{114}$ Despite the limited and inconsistent literature of mismatch repair deficiency/microsatellite instability in pancreatic cancer, a comparatively better prognosis and significantly prolonged survival for microsatellite instability high (MSI-H) compared to low (MSI-L) pancreatic cancer patients has been demonstrated. ${ }^{122-126}$ Several studies have evaluated the expression of PD-L1 in PDAC. ${ }^{14,127-131}$ As presented in a meta- 
analysis of nine studies, PD-L1 positive rate was found in $19 \%$ to $62.5 \%$ of PDAC patients. ${ }^{132}$ Although an association between checkpoint inhibitors PD-L1 and dMMR/MSI-H as well as with tumor mutational load (TML) has not been extensively studied, PD-L1 expression was seen in 38.9\% of dMMR tumors and 15.2\% of MMR proficient tumors, ${ }^{133,134}$ emerging with the potential role of MSI-H status as predictive of a response to immunotherapy. TML express the total number of somatic/acquired mutations per coding area of a tumor genome. ${ }^{135}$ It is associated with increased sensitivity to immunotherapy ${ }^{135}$ and MSI-H tumors. ${ }^{133}$ The clinical benefit of anti-programmed death-1 therapy with pembrolizumab has been evaluated in dMMR/MSI-H previously treated unresectable or metastatic noncolorectal cancer patients. ${ }^{136}$ Among 233 enrolled patients with MSI-H/dMMR, advanced pancreatic cancer has been the most common, followed by endometrial cancer, gastric cancer and cholangiocarcinoma. PFS and OS was evaluated at 2.1 (95\% CI 1.9-3.4) and 4.0 (95\% CI 2.1-9.8) months respectively. ${ }^{136}$ Currently there are several clinical trials conducted in MSI-high pancreatic ductal adenocarcinoma patients in combination with chemotherapy or immunotherapy agents, nevertheless no result have been posted yet (Table 4).

Homologous recombination deficiency (HRD) represents a prominent new biomarker in metastatic PDAC. Patients with HRD positive tumors are characterized by deregulation of DNA repair enzymes which makes these tumors highly susceptible to DNA damaging chemotherapy drugs, such as platinum derivatives, alkylating agents, and mitomycin C. ${ }^{137}$ Patients with HRD are also highly-sensitive to PARP inhibitors, as demonstrated by numerous trials in ovarian cancer,

Table 4 Clinical Trials in Pancreatic Cancer Patients with High Microsatellite Instability

\begin{tabular}{|c|c|c|c|c|c|c|}
\hline Intervention & & Cancer Stage & $\begin{array}{l}\text { Clinical } \\
\text { Phase/ } \\
\text { Identifier }\end{array}$ & $\begin{array}{l}\text { Outcomes: } \\
\text { Disease Free } \\
\text { Survival (Months) } \\
(95 \% \mathrm{Cl})\end{array}$ & $\begin{array}{l}\text { Outcomes: Median } \\
\text { Overall Survival } \\
\text { (Months) }(95 \% \mathrm{Cl})\end{array}$ & Reference \\
\hline Pembrolizumab & PD-I inhibitor & $\begin{array}{l}\text { MSI High/advanced } \\
\text { pancreatic cancer }\end{array}$ & $\begin{array}{l}\text { Phase II, } \\
\text { NCT02628067 }\end{array}$ & $\begin{array}{l}\text { Recruiting/2.1 } \\
(1.9-3.4)\end{array}$ & $\begin{array}{l}\text { I395 participants } 4.0 \\
(2.1-9.8)\end{array}$ & $\begin{array}{l}\text { Marabelle } \\
\text { et al } 2020^{136}\end{array}$ \\
\hline $\begin{array}{l}\text { Pembrolizumab/ } \\
\text { Sonidegib }\end{array}$ & $\begin{array}{l}\text { PD-I inhibitor, Hh-pathway } \\
\text { inhibitor }\end{array}$ & $\begin{array}{l}\text { MSI High/ advanced } \\
\text { pancreatic cancer }\end{array}$ & $\begin{array}{l}\text { Phase I, } \\
\text { NCT04007744 }\end{array}$ & Recruiting & Recruiting & $\begin{array}{l}\text { Clinic and } \\
\text { Institute } \\
2020^{152}\end{array}$ \\
\hline $\begin{array}{l}\text { Pembrolizumab/ } \\
\text { DEBIOII43 }\end{array}$ & PD-I inhibitor, IAP inhibitor & $\begin{array}{l}\text { Non-MSI-high/ } \\
\text { advanced/metastatic }\end{array}$ & $\begin{array}{l}\text { Phase I, } \\
\text { NCT0387I959 }\end{array}$ & Recruiting & Recruiting & $\begin{array}{l}\text { Berard et al } \\
2019^{153}\end{array}$ \\
\hline $\begin{array}{l}\text { Pembrolizumab/ } \\
\mathrm{XmAb}^{\circledR} 22841\end{array}$ & $\begin{array}{l}\text { PD-I inhibitor, CTLA-4/ } \\
\text { LAG-3 inhibitor }\end{array}$ & $\begin{array}{l}\text { MSI High/advanced or } \\
\text { metastatic pancreatic } \\
\text { cancer }\end{array}$ & $\begin{array}{l}\text { Phase I, } \\
\text { NCT03849469 }\end{array}$ & Recruiting & Recruiting & $\begin{array}{l}\text { Xencor and } \\
\text { Research } \\
2019^{154}\end{array}$ \\
\hline $\begin{array}{l}\text { Nivolumab/ } \\
\text { Ipilimumab/Radiation }\end{array}$ & $\begin{array}{l}\text { PD-I inhibitor, CTLA-4 } \\
\text { inhibitor, Radiation }\end{array}$ & $\begin{array}{l}\text { MSI High/pancreatic } \\
\text { adenocarcinoma }\end{array}$ & $\begin{array}{l}\text { Phase II, } \\
\text { NCT03104439 }\end{array}$ & Recruiting & Recruiting & $\begin{array}{l}\text { Hospital and } \\
\text { Squibb } \\
2017^{155}\end{array}$ \\
\hline $\begin{array}{l}\text { Nivolumab/ } \\
\text { Pembrolizumab/ } \\
\text { Atezolizumab/FT500/ } \\
\text { CY/Fludarabine }\end{array}$ & $\begin{array}{l}\text { PD-I inhibitor, PD-I } \\
\text { inhibitor, PD-LI inhibitor, } \\
\text { iPSC-NK cell, Cytotoxic } \\
\text { drugs }\end{array}$ & $\begin{array}{l}\text { MSI High/advanced } \\
\text { pancreatic cancer }\end{array}$ & $\begin{array}{l}\text { Phase I, } \\
\text { NCT0384III0 }\end{array}$ & Recruiting & Recruiting & $\begin{array}{l}\text { Therapeutics } \\
2019^{156}\end{array}$ \\
\hline $\begin{array}{l}\text { LY3300054/ } \\
\text { Ramucirumab/ } \\
\text { Abemaciclib/ } \\
\text { Merestinib/LY332/367 }\end{array}$ & $\begin{array}{l}\text { PD-I inhibitor, VEGFR2 } \\
\text { antagonist, CDK inhibitor, } \\
\text { HGFR inhibitor, anti-TIM3 }\end{array}$ & $\begin{array}{l}\text { MSI High/pancreatic } \\
\text { cancer }\end{array}$ & $\begin{array}{l}\text { Phase I, } \\
\text { NCT02791334 }\end{array}$ & Recruiting & Recruiting & $\begin{array}{l}\text { Lilly and } \\
\text { Company } \\
2016^{157}\end{array}$ \\
\hline $\begin{array}{l}\text { TIL } \\
\text { Fludarabine/CY }\end{array}$ & $\begin{array}{l}\text { Adoptive cell transfer } \\
\text { Cytotoxic drugs }\end{array}$ & $\begin{array}{l}\text { MSI High/advanced, } \\
\text { recurrent, or } \\
\text { metastatic pancreatic } \\
\text { cancer }\end{array}$ & $\begin{array}{l}\text { Phase II, } \\
\text { NCT03935893 }\end{array}$ & Recruiting & Recruiting & $\begin{array}{l}\text { Kammula } \\
2019^{158}\end{array}$ \\
\hline
\end{tabular}

Abbreviations: MSI, microsatellite instability; Hh-pathway, hedgehog signaling pathway; IAP, inhibitor of apoptosis proteins; VEGFR2, vascular endothelial growth factor receptor 2; CDK, cyclin-dependent kinase; HGFR, hepatocyte growth factor receptor; TIM3, T-cell immunoglobulin and mucin domain-3; TIL, tumor infiltrating lymphocytes; CY, Cyclophosphamide; iPSC-NK, induced pluripotent stem cells-derived Natural Killer cell. 
and to a lesser extent in other solid tumors, such as breast and prostate cancer. ${ }^{138}$ When it comes to PDAC, about $14-$ $44 \%$ of patients carry mutations in at least one HRD gene, with BRCA and ATM being the most frequent ${ }^{139}$ study. Moreover, the use of the PARP inhibitor olaparib as maintenance therapy after response to platinum-based chemotherapy in patients with BRCA-mutated metastatic PDAC has led to improved PFS over treatment with a placebo. ${ }^{140}$ Similarly, rucaparib, another PARP inhibitor has shown promising results as maintenance treatment in patients with mutations in BRCA1, BRCA2 and PALB2 in a phase II trial. ${ }^{141}$

These promising results in a malignancy associated with poor prognosis and scarce therapeutic options led to the investigation of PARP-based combination treatments. The synergy of immunotherapy and PARP inhibitors has been the subject of many preclinical studies which have generated encouraging results that, in turn, formed the base for many ongoing clinical trials in solid tumors. ${ }^{142}$ In PDAC, exploratory results from a Canadian phase II study showed improved clinical outcomes in patients with ATM mutated metastatic PDAC receiving a combination of durvalumab and tremelimumab with chemotherapy over those that received chemotherapy alone. The phase II POLAR trial is currently exploring the safety and efficacy of a combination of pembrolizumab and olaparib as maintenance treatment in patients with metastatic PDAC and HRD or major response to platinum-based chemotherapy NCT04666740. Another phase I/IIb trial is examining the combination of niraparib with either ipilimumab or nivolumab in a similar population NCT03404960.

Emerging molecular targets such as CXCR4, LAG-3 and TIGIT have been suggested as predictive biomarkers to immunotherapy response in preclinical studies and are currently explored in various solid tumors in combination with immunotherapy or other treatment modalities. ${ }^{143}$ CXCR4 has been associated with higher pro-inflammatory cytokine expression and with improved OS, suggesting a possible benefit with immunotherapy in CXCR4 high patients. ${ }^{144}$ There is also an ongoing phase II trial (NCT02826486) currently evaluating an CXCR4 inhibitor in metastatic PDAC. LAG-3 expression in tumor infiltrating T-cells was associated with worse DFS in a sample of 69 patients with resected PDAC. ${ }^{145}$ Data from pre-clinical models link CD155/TIGIT activity with immune evasion of pancreatic tumor cells. ${ }^{146}$ Finally, experimental immune checkpoints such as IDO, VISTA, TIM3 represent potential targets for novel checkpoint inhibitors and may lead to advancements in the barren field of pancreatic cancer immunotherapy. ${ }^{147}$

\section{Conclusion}

Regardless of recent advances in cancer treatment, pancreatic duct adenocarcinoma is associated with extremely poor prognosis, still remaining a challenging disease to treat. So far, surgical resection in combination with either adjuvant or neoadjuvant chemotherapy, depending on stage of the disease, offers modest improvement in survival rates. Nevertheless, the overall prognosis for patients of all stages combined remains poor. Although advances in immunotherapy have provided an effective and safe treatment regimen in several cancer types, results of clinical trials for pancreatic cancer treatment have uniformly been disappointing for the majority of these regimens when used as monotherapy. On the other hand, both neoadjuvant and adjuvant immunotherapy have shown promising results, so far, when combined with cytotoxic drugs and/or radiotherapy. It seems that neoadjuvant immunotherapy increases the presence of TILs in the PC microenvironment and reduces the apoptosis of naïve T cells as well as the suppression of T-cell proliferation caused by surgical stress. Moreover, tremendous improvements in our knowledge, concerning the complex molecular and cellular microenvironment of pancreatic cancer cells, have occurred during the last decade. This knowledge will probably lead to the identification of subgroups of patients who will gain more benefit from specific therapies, in accordance with the concept of personalized treatment. Identification of valid biomarkers may guide treatment options in a pathway of targeted therapies which, in combination with immunotherapy, may reduce toxicity and improve outcomes. Further clinical studies are important in order to overcome microenvironment resistance and enhance effective treatment.

\section{Disclosure}

The authors report no conflicts of interest in this work. 


\section{References}

1. Kleeff J, Korc M, Apte M, et al. Pancreatic cancer. Nat Rev Dis Primers. 2016;2:16022. doi:10.1038/nrdp.2016.22

2. Puckett Y, Garfield K. Pancreatic Cancer. StatPearls; 2021.

3. Sung H, Ferlay J, Siegel RL, et al. Global cancer statistics 2020: GLOBOCAN estimates of incidence and mortality worldwide for 36 cancers in 185 countries. CA Cancer J Clin. 2021;71(3):209-249. doi:10.3322/caac.21660

4. Rahib L, Smith BD, Aizenberg R, Rosenzweig AB, Fleshman JM, Matrisian LM. Projecting cancer incidence and deaths to 2030: the unexpected burden of thyroid, liver, and pancreas cancers in the United States. Cancer Res. 2014;74(11):2913-2921. doi:10.1158/0008-5472. CAN-14-0155

5. Siegel R, Miller K, Jemal A. Cancer statistics, 2015. CA Cancer J Clin. 2015;65(1):5e29.

6. Reyes-Gibby CC, Chan W, Abbruzzese JL, et al. Patterns of self-reported symptoms in pancreatic cancer patients receiving chemoradiation. J Pain Symptom Manage. 2007;34(3):244-252. doi:10.1016/j.jpainsymman.2006.11.007

7. Löhr M. Is it possible to survive pancreatic cancer? Nat Clin Pract Gastroenterol Hepatol. 2006;3(5):236-237. doi:10.1038/ncpgasthep0469

8. Herzberg B, Campo MJ, Gainor JF. Immune checkpoint inhibitors in non-small cell lung cancer. Oncologist. 2017;22(1):81-88. doi:10.1634/ theoncologist.2016-0189

9. Schizas D, Charalampakis N, Kole C, et al. Immunotherapy for pancreatic cancer: a 2020 update. Cancer Treat Rev. 2020;86:102016. doi:10.1016/j.ctrv.2020.102016

10. Hodi FS, O'Day SJ, McDermott DF, et al. Improved survival with ipilimumab in patients with metastatic melanoma. N Engl J Med. 2010;363 (8):711-723. doi:10.1056/NEJMoa1003466

11. Rosenberg SA. Decade in review-cancer immunotherapy: entering the mainstream of cancer treatment. Nat Rev Clin Oncol. 2014;11 (11):630-632. doi:10.1038/nrclinonc.2014.174

12. Pardoll DM. The blockade of immune checkpoints in cancer immunotherapy. Nat Rev Cancer. 2012;12(4):252-264. doi:10.1038/nrc3239

13. Disis ML. Mechanism of action of immunotherapy. Semin Oncol. 2014;41:S3-S13. doi:10.1053/j.seminoncol.2014.09.004

14. Nomi T, Sho M, Akahori T, et al. Clinical significance and therapeutic potential of the programmed death-1 ligand/programmed death-1 pathway in human pancreatic cancer. Clin Cancer Res. 2007;13(7):2151-2157. doi:10.1158/1078-0432.CCR-06-2746

15. Zhang Y, Velez-Delgado A, Mathew E, et al. Myeloid cells are required for PD-1/PD-L1 checkpoint activation and the establishment of an immunosuppressive environment in pancreatic cancer. Gut. 2017;66(1):124-136. doi:10.1136/gutjnl-2016-312078

16. Farren MR, Mace TA, Geyer S, et al. Systemic immune activity predicts overall survival in treatment-naive patients with metastatic pancreatic cancer. Clin Cancer Res. 2016;22(10):2565-2574. doi:10.1158/1078-0432.CCR-15-1732

17. Patel SP, Kurzrock R. PD-L1 expression as a predictive biomarker in cancer immunotherapy. Mol Cancer Ther. 2015;14(4):847-856. doi:10.1158/1535-7163.MCT-14-0983

18. Rice J, Ottensmeier CH, Stevenson FK. DNA vaccines: precision tools for activating effective immunity against cancer. Nat Rev Cancer. 2008;8 (2):108-120. doi:10.1038/nrc2326

19. Aranda F, Vacchelli E, Eggermont A, et al. Trial watch: peptide vaccines in cancer therapy. Oncoimmunology. 2013;2(12):e26621. doi:10.4161/ onci.26621

20. Vacchelli E, Martins I, Eggermont A, et al. Trial watch: peptide vaccines in cancer therapy. Oncoimmunology. 2012;1(9):1557-1576. doi:10.4161/onci.22428

21. Akce M, Zaidi MY, Waller EK, El-Rayes BF, Lesinski GB. The potential of CAR T cell therapy in pancreatic cancer. Front Immunol. 2018;9:2166. doi:10.3389/fimmu.2018.02166

22. Griffith JW, Sokol CL, Luster AD. Chemokines and chemokine receptors: positioning cells for host defense and immunity. Annu Rev Immunol. 2014;32:659-702. doi:10.1146/annurev-immunol-032713-120145

23. Dorr P, Westby M, Dobbs S, et al. Maraviroc (UK-427,857), a potent, orally bioavailable, and selective small-molecule inhibitor of chemokine receptor CCR5 with broad-spectrum anti-human immunodeficiency virus type 1 activity. Antimicrob Agents Chemother. 2005;49 (11):4721-4732. doi:10.1128/AAC.49.11.4721-4732.2005

24. Covino DA, Sabbatucci M, Fantuzzi L. The CCL2/CCR2 axis in the pathogenesis of HIV-1 infection: a new cellular target for therapy? Curr Drug Targets. 2016;17(1):76-110. doi:10.2174/138945011701151217110917

25. Fei L, Ren X, Yu H, Zhan Y. Targeting the CCL2/CCR2 axis in cancer immunotherapy: one stone, three birds? Front Immunol. 2021;12:771210. doi:10.3389/fimmu.2021.771210

26. Royal RE, Levy C, Turner K, et al. Phase 2 trial of single agent Ipilimumab (anti-CTLA-4) for locally advanced or metastatic pancreatic adenocarcinoma. J Immunother. 2010;33(8):828-833. doi:10.1097/CJI.0b013e3181eec14c

27. AstraZeneca. Study of tremelimumab in patients with advanced solid tumors. Available from: https://clinicaltrials.gov/show/NCT02527434. Accessed May 3, 2021.

28. Klaiber U, Hackert T, Neoptolemos JP. Adjuvant treatment for pancreatic cancer. Transl Gastroenterol Hepatol. 2019;4:27. doi:10.21037/ $\operatorname{tgh} .2019 .04 .04$

29. Okusaka T, Nakamura M, Yoshida M, et al. Clinical practice guidelines for pancreatic cancer 2019 from the Japan Pancreas Society: a synopsis. Pancreas. 2020;49(3):326-335. doi:10.1097/MPA.0000000000001513

30. Kayahara M, Funaki K, Tajima H, et al. Surgical implication of micrometastasis for pancreatic cancer. Pancreas. 2010;39(6):884-888. doi:10.1097/MPA.0b013e3181ce6daa

31. Trimble EL, Ungerleider RS, Abrams JA, et al. Neoadjuvant therapy in cancer treatment. Cancer. 1993;72(11 Suppl):3515-3524.

32. Byrne KT, Vonderheide RH. CD40 stimulation obviates innate sensors and drives T cell immunity in cancer. Cell Rep. 2016;15(12):2719-2732. doi:10.1016/j.celrep.2016.05.058

33. Amin S, Baine M, Meza J, Lin C. The impact of neoadjuvant and adjuvant immunotherapy on the survival of pancreatic cancer patients: a retrospective analysis. BMC Cancer. 2020;20(1):538. doi:10.1186/s12885-020-07016-8

34. Teng F, Mu D, Meng X, et al. Tumor infiltrating lymphocytes (TILs) before and after neoadjuvant chemoradiotherapy and its clinical utility for rectal cancer. Am J Cancer Res. 2015;5(6):2064-2074. 
35. Ogawa K, Hirai M, Katsube T, et al. Suppression of cellular immunity by surgical stress. Surgery. 2000;127(3):329-336. doi:10.1067/ msy.2000.103498

36. Hogan BV, Peter MB, Shenoy HG, Horgan K, Hughes TA. Surgery induced immunosuppression. Surgeon. 2011;9(1):38-43. doi:10.1016/j. surge.2010.07.011

37. Rahma OE, Katz MHG, Wolpin BM, et al. Randomized multicenter phase Ib/II study of neoadjuvant chemoradiation therapy (CRT) alone or in combination with pembrolizumab in patients with resectable or borderline resectable pancreatic cancer. J Clin Oncol. 2021;39(15_suppl):4128. doi:10.1200/JCO.2021.39.15_suppl.4128

38. University Z. Combination of anti-PD-1 antibody and chemotherapy in pancreatic cancer; 2019. Available from: https://clinicaltrials.gov/show/ NCT03983057. Accessed February 14, 2022.

39. Zhou X, Hou W, Gao L, Shui L, Yi C, Zhu H. Synergies of antiangiogenic therapy and immune checkpoint blockade in renal cell carcinoma: from theoretical background to clinical reality. Front Oncol. 2020;10:1321. doi:10.3389/fonc.2020.01321

40. Murphy JM, Rodriguez YAR, Jeong K, Ahn EE, Lim SS. Targeting focal adhesion kinase in cancer cells and the tumor microenvironment. Exp Mol Med. 2020;52(6):877-886. doi:10.1038/s12276-020-0447-4

41. Hopkins SK, Sharp M, Corp D, Verastem I. Study of pembrolizumab with or without defactinib following chemotherapy as a neoadjuvant and adjuvant treatment for resectable pancreatic ductal adenocarcinoma. Available from: https://clinicaltrials.gov/show/NCT03727880. Accessed March 26, 2021.

42. Saxena M, van der Burg SH, Melief CJM, Bhardwaj N. Therapeutic cancer vaccines. Nat Rev Cancer. 2021;21(6):360-378. doi:10.1038/ s41568-021-00346-0

43. Rosenberg A, Mahalingam D. Immunotherapy in pancreatic adenocarcinoma-overcoming barriers to response. J Gastrointest Oncol. 2018;9 (1):143-159. doi:10.21037/jgo.2018.01.13

44. Hopkins SK, Institute NC. Vaccine therapy with or without cyclophosphamide in treating patients undergoing chemotherapy and radiation therapy for stage I or stage II pancreatic cancer that can be removed by surgery. Available from: https://clinicaltrials.gov/show/NCT00727441. Accessed February 25, 2020.

45. Lee V, Ding D, Rodriguez C, et al. A phase 2 study of cyclophosphamide (CY), GVAX, pembrolizumab (Pembro), and stereotactic body radiation (SBRT) in patients (pts) with locally advanced pancreas cancer (LAPC). J Clin Oncol. 2021;39(suppl 15):abstr 4134. doi:10.1200/ JCO.2021.39.15_suppl.4134

46. Lee V, Rodriguez C, Shupe EM, et al. Phase II study of GM-CSF secreting allogeneic pancreatic cancer vaccine (GVAX) with PD-1 blockade antibody and stereotactic body radiation therapy (SBRT) for locally advanced pancreas cancer (LAPC). J Clin Oncol. 2017;35(15_suppl): TPS4154. doi:10.1200/JCO.2017.35.15_suppl.TPS4154

47. Hopkins SK, Sharp M, Corp D, Lilly E. Pilot study with CY, pembrolizumab, GVAX, and IMC-CS4 (LY3022855) in patients with borderline resectable adenocarcinoma of the pancreas. Available from: https://clinicaltrials.gov/show/NCT03153410. Accessed March 30, 2021.

48. Institute NC, Center NIoHC. Immune checkpoint inhibitor M7824 and the immunocytokine M9241 in combination with stereotactic body radiation therapy (SBRT) in adults with advanced pancreas cancer. Available from: https://clinicaltrials.gov/show/NCT04327986. Accessed June 18, 2021.

49. Corporation NG, Pharma L. Immunotherapy and SBRT study in borderline resectable pancreatic cancer. Available from: https://clinicaltrials. gov/show/NCT02405585. Accessed May 28, 2020.

50. Corporation NG, Pharma L. Immunotherapy study in borderline resectable or locally advanced unresectable pancreatic cancer. Available from: https://clinicaltrials.gov/show/NCT01836432. Accessed May 28, 2020.

51. Hopkins SK, Institute NC, Squibb B-M. Neoadjuvant/adjuvant GVAX pancreas vaccine (with CY) with or without nivolumab and urelumab trial for surgically resectable pancreatic cancer. Available from: https://clinicaltrials.gov/show/NCT02451982. Accessed February 14, 2022.

52. Center JCC, Squibb B-M, Ltd. N. Nivolumab in combination with chemotherapy before surgery in treating patients with borderline resectable pancreatic cancer. Available from: https://clinicaltrials.gov/show/NCT03970252. Accessed May 14, 2021.

53. Candel Therapeutics I, University OS. Neoadjuvant GMCI plus chemoradiation for advanced non-metastatic pancreatic adenocarcinoma. Available from: https://clinicaltrials.gov/show/NCT02446093. Accessed December 7, 2020.

54. Hopkins SK, Squibb BM. Trial of neoadjuvant and adjuvant nivolumab and BMS-813160 with or without GVAX for locally advanced pancreatic ductal adenocarcinomas. Available from: https://clinicaltrials.gov/show/NCT03767582. Accessed March 26, 2021.

55. ChemoCentryx. Phase 1b study of CCX872-B in patients with pancreatic adenocarcinoma. Available from: https://clinicaltrials.gov/show/ NCT02345408. Accessed August 16, 2019.

56. Katz A, Rochester UO. Pre-operative stereotactic body radiation therapy for pancreatic adenocarcinoma with or without CCX872-B. Available from: https://clinicaltrials.gov/show/NCT03778879. Accessed July 19, 2019.

57. Medicine WUSo, Institute NC. FOLFIRINOX plus PF-04136309 in patients with borderline resectable and locally advanced pancreatic adenocarcinoma. Available from: https://clinicaltrials.gov/show/NCT01413022. Accessed September 19, 2016.

58. Nywening TM, Wang-Gillam A, Sanford DE, et al. Targeting tumour-associated macrophages with CCR2 inhibition in combination with FOLFIRINOX in patients with borderline resectable and locally advanced pancreatic cancer: a single-centre, open-label, dose-finding, non-randomised, phase 1b trial. Lancet Oncol. 2016;17(5):651-662. doi:10.1016/S1470-2045(16)00078-4

59. Neoptolemos JP, Kleeff J, Michl P, Costello E, Greenhalf W, Palmer DH. Therapeutic developments in pancreatic cancer: current and future perspectives. Nat Rev Gastroenterol Hepatol. 2018;15(6):333-348. doi:10.1038/s41575-018-0005-x

60. Lutz E, Yeo CJ, Lillemoe KD, et al. A lethally irradiated allogeneic granulocyte-macrophage colony stimulating factor-secreting tumor vaccine for pancreatic adenocarcinoma. A phase II trial of safety, efficacy, and immune activation. Ann Surg. 2011;253(2):328-335. doi:10.1097/ SLA.0b013e3181fd271c

61. Jaffee EM, Hruban RH, Biedrzycki B, et al. Novel allogeneic granulocyte-macrophage colony-stimulating factor-secreting tumor vaccine for pancreatic cancer: a phase I trial of safety and immune activation. J Clin Oncol. 2001;19(1):145-156. doi:10.1200/JCO.2001.19.1.145

62. Hardacre JM, Mulcahy M, Small W, et al. Addition of algenpantucel-L immunotherapy to standard adjuvant therapy for pancreatic cancer: a phase 2 study. J Gastrointest Surg. 2013;17(1):94-100;discussion p 100-1. doi:10.1007/s11605-012-2064-6 
63. Hewitt DB, Nissen N, Hatoum H, et al. A phase 3 randomized clinical trial of chemotherapy with or without algenpantucel-L (hyperacute-pancreas) immunotherapy in subjects with borderline resectable or locally advanced unresectable pancreatic cancer. Ann Surg. 2020;271:1072-1079. doi:10.1097/SLA.0000000000004669

64. McCormick KA, Coveler AL, Rossi GR, Vahanian NN, Link C, Chiorean EG. Pancreatic cancer: update on immunotherapies and algenpantucel-L. Hum Vaccin Immunother. 2016;12(3):563-575. doi:10.1080/21645515.2015.1093264

65. Zeitouni D, Pylayeva-Gupta Y, Der CJ, Bryant KL. KRAS mutant pancreatic cancer: no lone path to an effective treatment. Cancers (Basel). 2016;8(4):45. doi:10.3390/cancers8040045

66. Gjertsen MK, Buanes T, Rosseland AR, et al. Intradermal ras peptide vaccination with granulocyte-macrophage colony-stimulating factor as adjuvant: clinical and immunological responses in patients with pancreatic adenocarcinoma. Int J Cancer. 2001;92(3):441-450. doi:10.1002/ ijc. 1205

67. Abou-Alfa GK, Chapman PB, Feilchenfeldt J, et al. Targeting mutated K-ras in pancreatic adenocarcinoma using an adjuvant vaccine. Am J Clin Oncol. 2011;34(3):321-325. doi:10.1097/COC.0b013e3181e84b1f

68. ASA T. Antigen-specific cancer immunotherapy (TG01) and gemcitabine as adjuvant therapy in resected pancreatic cancer. Available from: https://clinicaltrials.gov/show/NCT02261714. Accessed May 14, 2020.

69. Muscarella P, Wilfong LS, Ross SB, et al. A randomized, placebo-controlled, double blind, multicenter phase II adjuvant trial of the efficacy, immunogenicity, and safety of GI-4000 plus gem versus gem alone in patients with resected pancreas cancer with activating RAS mutations/ survival and immunology analysis of the R1 subgroup. J Clin Oncol. 2012;30(15_suppl):e14501. doi:10.1200/jco.2012.30.15_suppl.e14501

70. Miyazawa M, Katsuda M, Maguchi H, et al. Phase II clinical trial using novel peptide cocktail vaccine as a postoperative adjuvant treatment for surgically resected pancreatic cancer patients. Int J Cancer. 2017;140(4):973-982. doi:10.1002/ijc.30510

71. Hopkins SK, Squibb BM. Trial of neoadjuvant and adjuvant nivolumab and BMS-813160 with or without GVAX for locally advanced pancreatic ductal adenocarcinomas. Available from: https://clinicaltrials.gov/show/NCT03767582. Accessed August 20, 2021.

72. Medicine WUSo, Squibb B-M, Hospital TFfB-J, Health NIo, Institute NC. BMS-813160 with nivolumab and gemcitabine and Nab-paclitaxel in borderline resectable and locally advanced pancreatic ductal adenocarcinoma (PDAC). Available from: https://clinicaltrials.gov/show/ NCT03496662. Accessed November 3, 2021.

73. University N, Center RHLC. Ipilimumab and gemcitabine hydrochloride in treating patients with stage III-IV or recurrent pancreatic cancer that cannot be removed by surgery. Available from: https://clinicaltrials.gov/show/NCT01473940. Accessed March 6, 2020.

74. Squibb BM. An investigational study of immunotherapy combinations with chemotherapy in patients with gastric or gastroesophageal junction (GEJ) cancers. Available from: https://clinicaltrials.gov/show/NCT03662659. Accessed August 27, 2020.

75. A study of nivolumab by itself or nivolumab combined with ipilimumab in patients with advanced or metastatic solid tumors. Available from: https://clinicaltrials.gov/show/NCT01928394. Accessed February 14, 2022.

76. Le DT, Lutz E, Uram JN, et al. Evaluation of ipilimumab in combination with allogeneic pancreatic tumor cells transfected with a GM-CSF gene in previously treated pancreatic cancer. J Immunother. 2013;36(7):382-389. doi:10.1097/CJI.0b013e31829fb7a2

77. Hopkins SKCCCaJ, Squibb B-M, Cancer SUT, Aduro Biotech I, Research AAfC, Foundation L. GVAX pancreas vaccine (with CY) and CRS-207 with or without nivolumab. Available from: https://clinicaltrials.gov/show/NCT02243371. Accessed April 6, 2021.

78. Aglietta M, Barone C, Sawyer MB, et al. A phase I dose escalation trial of tremelimumab (CP-675,206) in combination with gemcitabine in chemotherapy-naive patients with metastatic pancreatic cancer. Ann Oncol. 2014;25(9):1750-1755. doi:10.1093/annonc/mdu205

79. Weiss GJ, Blaydorn L, Beck J, et al. Correction to: phase Ib/II study of gemcitabine, nab-paclitaxel, and pembrolizumab in metastatic pancreatic adenocarcinoma. Invest New Drugs. 2019;37:797. doi:10.1007/s10637-019-00763-x

80. Weiss GJ, Blaydorn L, Beck J, et al. Phase Ib/II study of gemcitabine, nab-paclitaxel, and pembrolizumab in metastatic pancreatic adenocarcinoma. Invest New Drugs. 2018;36(1):96-102. doi:10.1007/s10637-017-0525-1

81. University Z. Combination of anti-PD-1 antibody and chemotherapy in metastatic pancreatic cancer. Available from: https://clinicaltrials.gov/ show/NCT03977272. Accessed December 11, 2019.

82. Cheng K, Lv WR, Li X, Tian B, Cao D. Toripalimab with nab-paclitaxel/gemcitabine as first-line treatment for advanced pancreatic adenocarcinoma: updated results of a single-arm, open-label, phase Ib/II clinical study. J Clin Oncol. 2021;39(suppl15):abstr e16213. doi:10.1200/JCO.2021.39.15_suppl.e16213

83. Zhu H, Sun X, Pan X, Wu P, Chen J. Sindilimab combined with nab-paclitaxel plus gemcitabine as first-line treatment for patients with advanced pancreatic cancer. J Clin Oncol. 2021;39(suppl 15):abstr e16257. doi:10.1200/JCO.2021.39.15_suppl.e16257

84. Suurs FV, Lub-de Hooge MN, de Vries EGE, de Groot DJA. A review of bispecific antibodies and antibody constructs in oncology and clinical challenges. Pharmacol Ther. 2019;201:103-119. doi:10.1016/j.pharmthera.2019.04.006

85. Jin D, Guo S, Zhang Y, et al. Efficacy and safety of KN046 plus nab-paclitaxel/gemcitabine as first-line treatment for unresectable locally advanced or metastatic pancreatic ductal adenocarcinoma (PDAC). J Clin Oncol. 2021;39(suppl15):abstr 4138. doi:10.1200/ JCO.2021.39.15_suppl.4138

86. Xie J, Chen L, Hua Y, Ii H, Meng Z. 982P - A phase II study of SHR-1701 plus famitinib for patients with previously treated advanced pancreatic cancer or biliary tract cancer. Ann Oncol. 2021;32(supp1_5):S829-S866. doi:10.1016/annonc/annonc705

87. Berraondo P, Sanmamed MF, Ochoa MC, et al. Cytokines in clinical cancer immunotherapy. Br J Cancer. 2019;120(1):6-15. doi:10.1038/ s41416-018-0328-y

88. Grell P, Lin CC, Milella M, et al. Phase II study of the anti-TGF- $\beta$ monoclonal antibody (mAb) NIS793 with and without the PD-1 inhibitor spartalizumab in combination with nab-paclitaxel/gemcitabine (NG) versus NG alone in patients (pts) with first-line metastatic pancreatic ductal adenocarcinoma (mPDAC). J Clin Oncol. 2021;39(supp115):abstr TPS4173.

89. Awada AH, Zematis M, Ochsenreither S, et al. 538P - nadunolimab (CAN04), a first-in-class monoclonal antibody against IL1RAP, in combination with chemotherapy in subjects with pancreatic cancer (PDAC) and non-small cell lung cancer (NSCLC). Ann Oncol. 2021;32 (suppl_5):S583-S620. doi:10.1016/annonc/annonc699

90. Hecht JR, Lonardi S, Bendell JC, et al. Randomized phase III study of FOLFOX alone and with pegilodecakin as second-line therapy in patients with metastatic pancreatic cancer (SEQUOIA). J Clin Oncol. 2020;38(4_suppl):637. doi:10.1200/JCO.2020.38.4_suppl.637 
91. Whiting C, Lutz E, Nair N, et al. Phase II, randomized study of GVAX pancreas and CRS-207 immunotherapy in patients with metastatic pancreatic cancer: clinical update on long term survival and biomarker correlates to overall survival. $J$ Clin Oncol. 2015;33(3_suppl):261. doi: $10.1200 /$ jco.2015.33.3_suppl.261

92. Le DT, Picozzi VJ, Ko A $\bar{H}$, et al. Results from a phase IIb, randomized, multicenter study of GVAX pancreas and CRS-207 compared with chemotherapy in adults with previously treated metastatic pancreatic adenocarcinoma (ECLIPSE study). Clin Cancer Res. 2019;25:5493-5502. doi:10.1158/1078-0432.CCR-18-2992

93. Asahara S, Takeda K, Yamao K, Maguchi H, Yamaue H. Phase I/II clinical trial using HLA-A24-restricted peptide vaccine derived from KIF20A for patients with advanced pancreatic cancer. $J$ Transl Med. 2013;11:291. doi:10.1186/1479-5876-11-291

94. Suzuki N, Hazama S, Iguchi H, et al. Phase II clinical trial of peptide cocktail therapy for patients with advanced pancreatic cancer: venus-PC study. Cancer Sci. 2017;108(1):73-80. doi:10.1111/cas.13113

95. Kaufman HL, Kim-Schulze S, Manson K, et al. Poxvirus-based vaccine therapy for patients with advanced pancreatic cancer. $J$ Transl Med. 2007;5:60. doi:10.1186/1479-5876-5-60

96. Middleton G, Silcocks P, Cox T, et al. Gemcitabine and capecitabine with or without telomerase peptide vaccine GV1001 in patients with locally advanced or metastatic pancreatic cancer (TeloVac): an open-label, randomised, phase 3 trial. Lancet Oncol. 2014;15(8):829-840. doi:10.1016/S1470-2045(14)70236-0

97. Jiang N, Qiao G, Wang X, et al. Dendritic cell/cytokine-induced killer cell immunotherapy combined with S-1 in patients with advanced pancreatic cancer: a prospective study. Clin Cancer Res. 2017;23(17):5066-5073. doi:10.1158/1078-0432.CCR-17-0492

98. Feins S, Kong W, Williams EF, Milone MC, Fraietta JA. An introduction to chimeric antigen receptor (CAR) T-cell immunotherapy for human cancer. Am J Hematol. 2019;94(S1):S3-S9. doi:10.1002/ajh.25418

99. Raj D, Yang MH, Rodgers D, et al. Switchable CAR-T cells mediate remission in metastatic pancreatic ductal adenocarcinoma. Gut. 2019;68 (6):1052-1064. doi:10.1136/gutjnl-2018-316595

100. Chi J, Patel R, Rehman H, Goyal S, Saif MW. Recent advances in immunotherapy for pancreatic cancer. $J$ Cancer Metast Treat. $2020 ; 6: 43$. doi:10.20517/2394-4722.2020.90

101. Beatty GL, O'Hara MH, Lacey SF, et al. Activity of mesothelin-specific chimeric antigen receptor T cells against pancreatic carcinoma metastases in a phase 1 trial. Gastroenterology. 2018;155(1):29-32. doi:10.1053/j.gastro.2018.03.029

102. O'Hara MH, O'Reilly EM, Wolff RA. Gemcitabine (Gem) and nab-paclitaxel (NP) \pm nivolumab (nivo) \pm CD40 agonistic monoclonal antibody APX005M (Sotigalimab), in patients (Pts) with untreated metastatic pancreatic adenocarcinoma (mPDAC): phase ( $\mathrm{Ph}$ ) 2 final results. J Clin Oncol. 2021;39(suppl15):abstr 4019. doi:10.1200/JCO.2021.39.15_suppl.4019

103. Dubreuil P, Letard S, Ciufolini M, et al. Masitinib (AB1010), a potent and selective tyrosine kinase inhibitor targeting KIT. PLoS One. 2009;4 (9):e7258. doi:10.1371/journal.pone.0007258

104. Ezenfis J, Hermine O, Group AS. Masitinib plus gemcitabine as first-line treatment of pancreatic cancer with pain: results from phase 3 study AB12005. J Clin Oncol. 2021;39(15_suppl):4018. doi:10.1200/JCO.2021.39.15_suppl.4018

105. Cassier PA, Garin G, Eberst L, et al. MEDIPLEX: a phase 1 study of durvalumab (D) combined with pexidartinib (P) in patients (pts) with advanced pancreatic ductal adenocarcinoma (PDAC) and colorectal cancer (CRC). J Clin Oncol. 2019;37(15_suppl):2579. doi:10.1200/ JCO.2019.37.15_suppl.2579

106. Wang Y, Deng W, Li N, et al. Combining immunotherapy and radiotherapy for cancer treatment: current challenges and future directions. Front Pharmacol. 2018;9:185. doi:10.3389/fphar.2018.00185

107. Seery TE, Nangia CS, Sender LS, Reddy SK, Soon-Shiong P. Trial in progress: open-label, randomized, comparative phase $2 / 3$ study of combination immunotherapy plus standard-of-care chemotherapy and SBRT versus standard-of-care chemotherapy for the treatment of locally advanced or metastatic pancreatic cancer. $J$ Clin Oncol. 2021;39(suppl15):abstr TPS4174. doi:10.1200/JCO.2021.39.15_suppl.TPS4174

108. Li Z, Li Q, Ma Z, et al. Camrelizumab combined with ablation and chemotherapy for pancreatic cancer with liver metastases: a single-arm, phase II, prospective clinical study. J Clin Oncol. 2021;39(suppl15):abstr TPS4169. doi:10.1200/JCO.2021.39.15_suppl.TPS4169

109. Neesse A, Algul H, Tuveson DA, Gress TM. Stromal biology and therapy in pancreatic cancer: a changing paradigm. Gut. 2015;64 (9):1476-1484. doi:10.1136/gutjnl-2015-309304

110. Torphy RJ, Zhu Y, Schulick RD. Immunotherapy for pancreatic cancer: barriers and breakthroughs. Ann Gastroenterol Surg. 2018;2 (4):274-281. doi:10.1002/ags3.12176

111. Murakami T, Hiroshima Y, Matsuyama R, Homma Y, Hoffman RM, Endo I. Role of the tumor microenvironment in pancreatic cancer. Ann Gastroenterol Surg. 2019;3(2):130-137. doi:10.1002/ags3.12225

112. Vignali DA, Collison LW, Workman CJ. How regulatory T cells work. Nat Rev Immunol. 2008;8(7):523-532. doi:10.1038/nri2343

114. Bailey P, Chang DK, Forget MA, et al. Exploiting the neoantigen landscape for immunotherapy of pancreatic ductal adenocarcinoma. Sci Rep. 2016;6:35848. doi: $10.1038 /$ srep35848

115. Noy R, Pollard JW. Tumor-associated macrophages: from mechanisms to therapy. Immunity. 2014;41(1):49-61. doi:10.1016/j. immuni.2014.06.010

116. Allavena P, Piemonti L, Longoni D, et al. IL-10 prevents the differentiation of monocytes to dendritic cells but promotes their maturation to macrophages. Eur J Immunol. 1998;28(1):359-369. doi:10.1002/(SICI)1521-4141(199801)28:01<359::AID-IMMU359>3.0.CO;2-4

117. Blazquez JL, Benyamine A, Pasero C, Olive D. New insights into the regulation of gammadelta $\mathrm{T}$ cells by BTN3A and other BTN/BTNL in tumor immunity. Front Immunol. 2018;9:1601. doi:10.3389/fimmu.2018.01601

118. Le DT, Durham JN, Smith KN, et al. Mismatch repair deficiency predicts response of solid tumors to PD-1 blockade. Science. 2017;357 (6349):409-413. doi:10.1126/science.aan6733

119. Yamamoto H, Imai K. Microsatellite instability: an update. Arch Toxicol. 2015;89(6):899-921. doi:10.1007/s00204-015-1474-0

120. Lower SS, McGurk MP, Clark AG, Barbash DA. Satellite DNA evolution: old ideas, new approaches. Curr Opin Genet Dev. 2018;49:70-78. doi:10.1016/j.gde.2018.03.003

121. Veigl ML, Kasturi L, Olechnowicz J, et al. Biallelic inactivation of hMLH1 by epigenetic gene silencing, a novel mechanism causing human MSI cancers. Proc Natl Acad Sci U S A. 1998;95:8698-8702. doi:10.1073/pnas.95.15.8698 
122. Hu ZI, Shia J, Stadler ZK, et al. Evaluating mismatch repair deficiency in pancreatic adenocarcinoma: challenges and recommendations. Clin Cancer Res. 2018;24(6):1326-1336. doi:10.1158/1078-0432.CCR-17-3099

123. Nakata B, Wang YQ, Yashiro M, et al. Prognostic value of microsatellite instability in resectable pancreatic cancer. Clin Cancer Res. 2002;8 (8):2536-2540.

124. Fraune C, Burandt E, Simon R, et al. MMR deficiency is homogeneous in pancreatic carcinoma and associated with high density of cd8-positive lymphocytes. Ann Surg Oncol. 2020;27:3997-4006. doi:10.1245/s10434-020-08209-y

125. Yamamoto H, Itoh F, Nakamura H, et al. Genetic and clinical features of human pancreatic ductal adenocarcinomas with widespread microsatellite instability. Cancer Res. 2001;61(7):3139-3144.

126. Eatrides JM, Coppola D, Diffalha SA, Kim RD, Springett GM, Mahipal A. Microsatellite instability in pancreatic cancer. J Clin Oncol. 2016;34 (15_suppl):e15753. doi:10.1200/JCO.2016.34.15_suppl.e15753

127. Wang L, Ma Q, Chen X, Guo K, Li J, Zhang M. Clinical significance of B7-H1 and B7-1 expressions in pancreatic carcinoma. World J Surg. 2010;34(5):1059-1065. doi:10.1007/s00268-010-0448-X

128. Chen Y, Sun J, Zhao H, et al. The coexpression and clinical significance of costimulatory molecules B7-H1, B7-H3, and B7-H4 in human pancreatic cancer. Onco Targets Ther. 2014;7:1465-1472. doi:10.2147/OTT.S66809

129. Loos M, Giese NA, Kleeff J, et al. Clinical significance and regulation of the costimulatory molecule B7-H1 in pancreatic cancer. Cancer Lett. 2008;268(1):98-109. doi:10.1016/j.canlet.2008.03.056

130. Geng L, Huang D, Liu J, et al. B7-H1 up-regulated expression in human pancreatic carcinoma tissue associates with tumor progression. $J$ Cancer Res Clin Oncol. 2008;134(9):1021-1027. doi:10.1007/s00432-008-0364-8

131. Birnbaum DJ, Finetti P, Lopresti A, et al. Prognostic value of PDL1 expression in pancreatic cancer. Oncotarget. 2016;7(44):71198-71210. doi:10.18632/oncotarget.11685

132. Gao HL, Liu L, Qi ZH, et al. The clinicopathological and prognostic significance of PD-L1 expression in pancreatic cancer: a meta-analysis. Hepatobiliary Pancreat Dis Int. 2018;17(2):95-100. doi:10.1016/j.hbpd.2018.03.007

133. Salem ME, Puccini A, Grothey A, et al. Landscape of tumor mutation load, mismatch repair deficiency, and PD-L1 expression in a large patient cohort of gastrointestinal cancers. Mol Cancer Res. 2018;16(5):805-812. doi:10.1158/1541-7786.MCR-17-0735

134. Kim ST, Klempner SJ, Park SH, et al. Correlating programmed death ligand 1 (PD-L1) expression, mismatch repair deficiency, and outcomes across tumor types: implications for immunotherapy. Oncotarget. 2017;8(44):77415-77423. doi:10.18632/oncotarget.20492

135. Champiat S, Ferte C, Lebel-Binay S, Eggermont A, Soria JC. Exomics and immunogenics: bridging mutational load and immune checkpoints efficacy. Oncoimmunology. 2014;3(1):e27817. doi:10.4161/onci.27817

136. Marabelle A, Le DT, Ascierto PA, et al. Efficacy of pembrolizumab in patients with noncolorectal high microsatellite instability/mismatch repair-deficient cancer: results from the phase II KEYNOTE-158 study. J Clin Oncol. 2020;38(1):1-10. doi:10.1200/jco.19.02105

137. Pokataev I, Fedyanin M, Polyanskaya E, et al. Efficacy of platinum-based chemotherapy and prognosis of patients with pancreatic cancer with homologous recombination deficiency: comparative analysis of published clinical studies. ESMO Open. 2020;5(1):e000578. doi:10.1136/ esmoopen-2019-000578

138. Nguyen L, W. M. Martens J, Van Hoeck A, Cuppen E. Pan-cancer landscape of homologous recombination deficiency. Nat Commun. 2020;11 (1):5584. doi:10.1038/s41467-020-19406-4

139. Casolino R, Paiella S, Azzolina D, et al. Homologous recombination deficiency in pancreatic cancer: a systematic review and prevalence meta-analysis. J Clin Oncol. 2021;39(23):2617-2631. doi:10.1200/jco.20.03238

140. Golan T, Hammel P, Reni M, et al. Maintenance olaparib for germline BRCA-mutated metastatic pancreatic cancer. $N$ Engl $J$ Med. 2019;381 (4):317-327. doi:10.1056/NEJMoa1903387

141. Reiss KA, Mick R, O’Hara MH, et al. Phase II study of maintenance rucaparib in patients with platinum-sensitive advanced pancreatic cancer and a pathogenic germline or somatic variant in BRCA1, BRCA2, or PALB2. J Clin Oncol. 2021;39(22):2497-2505. doi:10.1200/ JCO.21.00003

142. Vikas P, Borcherding N, Chennamadhavuni A, Garje R. Therapeutic potential of combining PARP inhibitor and immunotherapy in solid tumors. Front Oncol. 2020;10:570. doi:10.3389/fonc.2020.00570

143. Shan C, Li X, Zhang J. Progress of immune checkpoint LAG-3 in immunotherapy. Oncol Lett. 2020;20(5):207. doi:10.3892/ol.2020.12070

144. Seeber A, Kocher F, Pircher A, et al. High CXCR4 expression in pancreatic ductal adenocarcinoma as characterized by an inflammatory tumor phenotype with potential implications for an immunotherapeutic approach. J Clin Oncol. 2021;39(suppl15):abstr 4021. doi:10.1200/ JCO.2021.39.15_suppl.4021

145. Seifert L, Plesca I, Muller L, et al. LAG-3-expressing tumor-infiltrating t cells are associated with reduced disease-free survival in pancreatic cancer. Cancers (Basel). 2021;13(6):1297. doi:10.3390/cancers13061297

146. Freed-Pastor WA, Lambert LJ, Ely ZA, et al. The CD155/TIGIT axis promotes and maintains immune evasion in neoantigen-expressing pancreatic cancer. Cancer Cell. 2021;39:1342-1360.e14. doi:10.1016/j.ccell.2021.07.007

147. Popp FC, Capino I, Bartels J, et al. Expression of immune checkpoint regulators IDO, Vista, LAG3, and TIM3 in resected pancreatic ductal adenocarcinoma. Cancers (Basel). 2021;13(11):2689. doi:10.3390/cancers13112689

148. Group G-MOC, Immunotherapeutics O, Squibb B-M. Maintenance therapy with OSE2101 vaccine alone or in combination with nivolumab, or FOLFIRI after induction therapy with FOLFIRINOX in patients with locally advanced or metastatic pancreatic ductal adenocarcinoma. Available from: https://clinicaltrials.gov/show/NCT03806309. Accessed February 12, 2020.

149. Limited K, Sanofi. Safety and efficacy of KY1044 and atezolizumab in advanced cancer; 2019. Available from: https://clinicaltrials.gov/show/ NCT03829501. Accessed February 14, 2022

150. Mehrotra S, Britten CD, Chin S, et al. Vaccination with poly(IC:LC) and peptide-pulsed autologous dendritic cells in patients with pancreatic cancer. J Hematol Oncol. 2017;10(1):82. doi:10.1186/s13045-017-0459-2

151. Morphotek. An efficacy study of MORAb-009 in subjects with pancreatic cancer. Available from: https:/clinicaltrials.gov/show/NCT00570713. Accessed September 9, 2015.

152. Clinic M, Institute NC. Sonidegib and pembrolizumab in treating patients with advanced solid tumors. Available from: https:/clinicaltrials.gov/ show/NCT04007744. Accessed January 15, 2021. 
153. Berard CL, Sharp M, Corp D, Sa DI. Pembrolizumab in combination with debio 1143 in pancreatic and colorectal advanced/metastatic adenocarcinoma. Available from: https://clinicaltrials.gov/show/NCT03871959. Accessed May 10, 2021.

154. Xencor I, Research IC. A study of XmAb ${ }^{\circledR} 22841$ monotherapy \& in combination w/ pembrolizumab in subjects w/ selected advanced solid tumors. Available from: https://clinicaltrials.gov/show/NCT03849469. Accessed May 5, 2021.

155. Hospital MG, Squibb B-M. Nivolumab and ipilimumab and radiation therapy in MSS and MSI high colorectal and pancreatic cancer. Available from: https://clinicaltrials.gov/show/NCT03104439. Accessed March 25, 2021.

156. Therapeutics F. FT500 as monotherapy and in combination with immune checkpoint inhibitors in subjects with advanced solid tumors. Available from: https://clinicaltrials.gov/show/NCT03841110. Accessed February 14, 2022.

157. Lilly and Company. A study of anti-PD-L1 checkpoint antibody (LY3300054) alone and in combination in participants with advanced refractory solid tumors. Available from: https://clinicaltrials.gov/show/NCT02791334. Accessed December 17, 2020.

158. Kammula U, Pittsburgh UO. Adoptive transfer of tumor infiltrating lymphocytes for advanced solid cancers. Available from: https://clinical trials.gov/show/NCT03935893. Accessed February 14, 2022.

\section{Publish your work in this journal}

Cancer Management and Research is an international, peer-reviewed open access journal focusing on cancer research and the optimal use of preventative and integrated treatment interventions to achieve improved outcomes, enhanced survival and quality of life for the cancer patient. The manuscript management system is completely online and includes a very quick and fair peer-review system, which is all easy to use. Visit http://www.dovepress.com/testimonials.php to read real quotes from published authors.

Submit your manuscript here: https://www.dovepress.com/cancer-management-and-research-journal 